\title{
LA POLITIQUE COLONIALE DE LA FRANCE AU DÉBUT DU SECOND EMPIRE
}

(INDO-CHINE, 1852- 1858)

PAR

HENRI CORDIER.

(Suite. $)^{1)}$

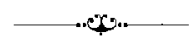

\section{CHAPITRE XVI.}

\section{La Situation en Annam.}

M. de Bourboulon dans une de ses dépêches ${ }^{2}$ ) au Ministre des Opinion de M. Affaires étrangères rappelant ce qu'il avait écrit en 1852 à la suite de Bourboulon. de l'assassinat de M. Bonnard ${ }^{3}$ ) au Tong-king, disait que pour que l'intervention de la France en Cochinchine fut fructueuse, deux conditions lui paraissaient indispensables: .

"La première que notre action à l'égard de cette contrée s'exerça isolément, et ne s'assoçia à celle d'aucune autre nation; la seconde que la démonstration, par laquelle on se proposerait d'établir de nouveaux rapports et de conclure un traité avec la Cochinchine fût une entreprise sérieuse, ne se bornât pas à une tentative de négociation ou à des actes d'hostilité passagers, mais fût poussée jusqu'au bout et suivie, surtout comme garantie pour l'avenir, de l'occupation permanente d'un point du territoire cochinchinois offrant une position maritime et militaire.

«D'après l'opinion que j’exprimais à cette époque, continuait notre Ministre, particulièrement en ce qui concerne la seconde de ces conditions, et cela en m'appuyant de l'expérience et de l'autorité de l'un des plus anciens et des plus distingués parmi nos missionnaires de la Cochinchine, V. E. concevra facilement que je n'aie pu considérer autrement que comme regrettable la mission qui fut confiée à M. de Montigny pour la Cochinchine vers le commencement de l'anncé dernière. En effet, sans prévoir tous les inconvénients qui devaient résulter des

1) Voir T'oung pao, 1909, Mars, Mai, Juillet et Décembre. - 1910, Juillet.

2) Macao, $1^{\text {er }}$ septembre 1857 .

3) Jean Louis Bonnard, du diocèse de Lyon; Missions étrangères de Paris; parti le 8 février 1849; missionnaire an Tong.king occidental; décapité le $I^{\text {er }}$ mai 1852 à Vi-hoang, âgé de 28 ans. 
longs retards apportés dans l'accomplissement de cette mission, de l'intervalle de près de quatre mois qui s'écoula entre l'apparition du Catinat dans les eaux de Tourane annonçant les demandes qu'un Plénipotentiaire français était chargé de venir présenter à la Cour de Hué, et l'arrivée de ce Plénipotentiaire lui-même, il était à peu près évident pour qui connaissait les antécédents de la question et la position des choses à ce moment, que la tentative qu'on allait faire pour renouer des rapports avec la Cochinchine ne pouvait avoir que bien peu de chances de succès.»

\section{M. de Bourboulon ajoutait que l'évènement u'ayant que trop justifié} ses prévisions, il se bornerait à indiquer brièrement les principales causes qui, à son sens, s'opposaient en priucipe à la réussite de cette entreprise telle qu'elle était conçue, et devant lesquelles, à part même les fautes commises dans l'exécution, devaient échouer les démarches de notre malencontreux négociateur:

«La première cause d'insuccès était sans doute daus les dispositions on ne peut moins favorables que des demandes telles que celles que M. de Montigny était chargé de présenter devaient rencontrer dans le Gouvernement cochinchinois: si l'on considère en effet, quels étaient nos rapports avec ce Gouvernement depuis 1847 (la destruction de la flotte cochinchinoise par le Commandant Lapierre), l'ombrageux système d'isolement et d'exclusion dans lequel, à l'exemple d'autres despotes de l'Extrême-Orient, il s'était renfermé depuis longtemps à l'égard des nations européennes, enfin l'accueil qui avait été fait l'année précédente aux ouvertures d'un Plénipotentiaire Anglais pour la conclusion d'un traité de commerce avec la Grande Bretagne, on concoit facilement que la Cour de Hué devait être très peu disposée à entrer en négociations avec une puissance dont elle était séparée par les plus fâcheux antécédents, qui en raison de ces antécédents devait Jui inspirer plus que toute autre de l'éloignement et de la crainte et qui se présentait avec des exigences les plus contraires, les plus antipathiques à ce qui avait été pendant ces 40 dernières années la base presque invariable de sa politique. Pour vaincre ces mauvaises dispositions, pour déterminer le Gouvernement Annamite à traiter avec nous et à accéder à nos demandes, il eût donc fallu être en mesure d'exercer sur lui Ja plus forte pression, de l'intimider en un mot, de lui imposer par la contrainte le traité que nous voulions en obtenir. Or, si je suis bien informé, les instructions données à M. de Montigny, tout en lui laissant peut-être la latitude d'agir sur le Gouvernement Cochinchinois par l'effet d'une menace éloignée en cas de refus, ne l'autorisaient pas à recourir immédiatement à l'emploi de la force; y eut-il été autorisé d'ailleurs, les quelques bâtiments mis à sa disposition ne présentaient 
pas une force suffisante pour pouvoir pousser très loin les mesures coercitives, dans le cas où ce Guuvernement prendrait comme il le prit en effet le parti de résister; leur action ne pouvait guère aller plus loin que de s'emparer des forts qui défendent l'entrée de la rivière de Hué, comme le Catinat quelques jours après son arrivée, avait réduit et désarmé ceux du port de Tourane et il est fort donteux que même cette démonstration, si elle avait été faite, aurait suff pour amener le roi Tu Duc à souscrire à nos demandes. Il faut ajouter enfin, que ces bátiments, momentanément détachés de la station de Chine, où le besoin de leur présence allait se faire bientôt vivement sentir, n'étaient affectés que pour un temps limité et pour ainsi dire en passant, au service de la mission dirigée sur la Cochinchine, sans beaucoup d'égard aux éventualités d'une négociation qui était de sa nature des plus délicates, de sorte que leur action en tous cas ne pouvait être que passagère tandis qu'il importait je crois au plus haut degré, quel que fut l'accueil fait aux premières demandes de nutre Plénipotentiaire et la tournure subséquente prise par la négociation, que le Gouvernement Cochinchinois fut convaincu par la présence permanente, n'eât-ce été que d'un seul de nos bâtiments de guerre devant Tourane, qu'il s'agissait d'une entreprise sérieuse et que notre ferme intention était d'y donner suite."

M. de Bourboulon continuait: que pour mettre le Ministre à même d'apprécier toutes les circonstances qui rendaient le succès de la Mission de M. de Montigny des plus chanceux et pour ainsi dire impossible, à moins qu'elle ne fut appuyée d'une puissante expédition et cela surtout au point de vue de ce qui constituait la partie la plus importante et la plus délicate de cette mission, la question religieuse, il lui demande la permission de placer un aperçu de la situation que présentait le royaume annamite environ un an avant l'époque où notre Plénipotentiaire fut chargé de se rendre en Cochiuchine, d'après des informations qui lui furent adressées par l'un des chefs de nos missions les plus expérimentés (Mgr. Rtoron) peu de temps avant son départ pour la France, vers le mois d'octobre 1855 .

«Depuis l'exécution de Mr. Bonnard, qui eut lieu en mai 1852, nos missions avaient joui d'une certaine tranquillité relative, lorqu'au commencement de 1854, une conspiration fut découverte ayant pour objet de détrôner le roi Tu 
Duc et de mettre à sa place son frère aîné Hoàng-bào 1), que l'influence d'un grand mandarin appelé Long Koue, qui a gouverné la Cochinchine pendant plus de 30 ans sous les règnes successifs de Minh-mang et de son fils Thiĉu-tri, et quí est encore aujourd'hui le premier Ministre et le principal Conseiller du roi Tu Duc, fils de Thiêu-tri, avait écarté en 1847 de la succession au trône annamite. Parmi les conspirateur's se trouvaient plusieurs mandarins dont quelques uns furent mis à mort et les autres envoyés en exil; le Prince Hoàng-bào luimême, chef et objet du complot devait être mis à mort, mais son frère lui fit grâce et se contenta de le faire sévèrement garder dans l'enceinte de sa résidence où il est resté depuís lors renfermé, se livrant, dit-on, à l'étude đes lettres et des sciences. La découverte de cette conspiration et la punition des coupables n'eurent cependant pas pour effet de rétablir le calme dans le royaume annamite, mais furent au contraire en quelque sorte le signal de vastes préparatifs pour une guerre civile qui éclata au commencement de l'année suivante (1855) et se répandit bientít sur plusieurs provinces du Tong-King et de la Basse Cochinchine. Je laisse parler à ce sujet Mgr. Retord.

"All Tong-King, écrivait-il, il y a déjà eu bien des fois des tentatives de «révolte, car tout le monde déteste ce Gouvernement corrupteur et corrompu «et tous aspirent à un changement sans savoir, hélas! s'ils rencontreront quelque «chose de meilleur. Mais jamais on ne s'était préparé à la guerre avec autant «d'ardeur et d'assurance que l'année dernière (1854). Partout, on s'organisait, «on s'exerçait, on forgeait des armes. Les rebelles se rassemblaient d'une pro«vince à l'autre; ils déflaient pendant la nuit et souvent même pendant le «jour par troupes considérables et s'établissaient dans différentes positions, au «milieu des montagnes, où ils formaient des camps que les Mandarins n'osaient "pas attaquer. Ils étaient, disait-on, très-nombreux, on les comptait par «50000; ces bandes étaient composées d'Annamites de la plaine, de sauvages «des montagnes, de Chinois émigr'és, de Laossiens du nord, de mandarins des«titués, de lettrés sans place, de débiteurs insolvables, de brigands poursuivís, «de voleurs émérites et de partisans de l'ancienne dynastie de Le, dont un «rejeton vrai ou fictif est ì leur tête."

«On pouvait croire que cette formidable insurrection allait affranchir d'un seul coup tout le Tong-King du joug annamite et s'avancer triomphante jusqu'aux portes de Hué, cependant il n'en fut rien. Les rebelles qui s'étaient mis en mouvement dans le courant de janvier et avaient commencé leurs opérations dans la plaine, après s'être emparés en quelques jours de plusieurs localités

1) «Thiệu-tri, lorsqu'il n'était que prince royal, avait épousé une fille de Gò-công, nommée $C \delta$ Hàng. Cette jeune fille fut présentée par sa tante, femme jeune encore, qui elle-même ne déplut point au prince. $\mathrm{La}$ tante et la nièce eurent chacune un fils. La tante donna le jour an prince Hoł̀ng-bà 0 , ou Au-phong, et ln nièce mit au monde quelque temps après le prince Iloàng-nhậm (Tư' d'u'c). Ce dernier fut choisi par Thiệu-tri pour lui succéder.» ('Tru'o'ng-vinh-ky, Cours a'hist. annamite, II, p. 272). 
importantes, de plusieurs arrondissements, et même, dit-on, d'une province toute entière, et après avoir mis plusieurs fois en fuite les mandarins avec leurs troupes, furent battus à leur tour au Fu-ly; quelques-uns ayant été pris, firent des révélations par suite desquelles leurs complices furent arrêtés et exécutés, ce qui jeta beaucoup de découragement parmi les insurgés; enfin le roi ayant envoyé des troupes de la Cochinchine, ils se débandèrent et se hatèrent de rentrer les uns dans les montagnes et les autres chez eux. Cependant les rebelles étaient loin d'être détruits; leurs plus fortes bandes n'étaient pas encore sorties de leur's retranchements dans les montagnes, et on s'attendait à les voir reparaître bientôt plus nombreux et plus redoutables que la première fois; j'ignore ce qui en est arrivé.

«Qu'en résulterait-il? dit à ce sujet Mgr. Retord, probablement ce qui est «résulté de leur première expédition; des massacres, des pillages et des incen«dies, c'est-à-dire le malheur du peuple et la dévastation du pays. En temps «de guerre, les mandarins ont un système de répression atroce. S'ils savent que «tel individu est parmi les rebelles, ne pouvant pas arrêter le coupable, ils «arrêtent ses parents et les dignitaires de son village et les exécutent à sa "place, ou mème quelquefois les relâchent, si on peut leur donner une somme «d'argent capable de satisfaire leur cupidité. Autre expédient aussi lâche "qu'inique: quand une troupe de rebelles passe ou séjourne quelques jours «dans un village trop faible pour leur résister, les mandarins attendent que «les rebelles soient partis pour venir ensuite brûler et saccager le pauvre «village par où ils ont passé; puis ils chantent victoire comme s'ils avaient mis «l'ennemi en fuite. C'est de cette manière et pour ce motif qu'ils ont incendié «et dévasté plusieur's villages. (lit il en cite deux ou trois). Dans ce dernier «village, ils coupèrent la tête à plusieurs individus dans leurs propres maisons. «Mais rien n'est horrible comme la ruse infernale inventée par les mandarins "pour convir leurs défaites lorsque les rebelles les ont battus on mis en fuite; «ils coupent les têtes aux gens des villages qu'ils traversent en fuyant, puis «ils coupent les oreilles et les nez de ces têtes d'hommes paisibles ou même de «femmes timides, en font comme des espèces de chapelets qu'ils envoient au "roi comme preuve palpable de leur victoire signalée, afin d'en obtenir les "récompenses et les avancements dûs à leur bravoure. Voilà ce que c'est que «la guerre civile ici." 1)

Ces tentatives de rébellion ont pour contre-coup comme en 1834, sous Minh-mang, lors de la rérolte de Khoi, une persécution sanglante des Chrétiens. M. de Bourboulou poursuit:

«On ne voit pas clairement par yuel calcul de politique le Gouvernement

1) Ce sont les mêmes moeurs administratives et la même manière de faire la guerre qu'en Chine, peut-être avec un degré de barbarie en plus. 
Annamite au milieu de ces agitations d'intérêts ambitieux et de passions révoJutionnaires ayant pour dernier résultat la guerre civile crut devoir redoubler de rigueurs contre la religion catholique, ses ministres et ses sectateurs, notoirement étrangers à tous ces mouvements autant, comme le dit Mgr. Retord par principe de religion que par prudence. 1). Nos missionnaires en diraient sans doute facilement la raison. C'est peut-être que ce Gouvernement dans sa haine contre «la religion de Jésus" croit trouver dans ces circonstances une occasion de la discréditer aux yeux du peuple, en affectant de lui attribuer une part, une sorte de complicité, dans tous les attentats et toutes les calamités publiques; quoi qu'il en soit, ce fut vers l'époque où la révolte dont j'ai parlée tout à l'heure venait d'être réprimée au Tong-King autant que je puis la discerner, au mois de mars ou d'avril 1855, que fut promulgué un nouvel Edit de persécution contre les Missionnaires étrangers et contre les Chrétiens, édit qui résumait et remettait en vigueur toutes les prohibitions et les pénalités les plus rigoureuses du règne de Minh-mang et de la première année du règne

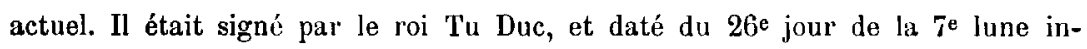
tercalaire de l'année précédente, c'est-à-dire du mois de Juin ou Juillet 1854.

«Comme ceì Edit, rédigé par les Ministres du Conseil reflète toute la politique du Gouvernement Annamite à l'égard de la religion catholique, il n'est peut-ître pas inutile d'en donner ici une courte analyse.

"L'édit même est précédé d'un préambule intitulé ordre royal qui commence ainsi: «La religion de Jésus vient des barbares d'Europe; elle employe l'image de Jésus crucifié pour séduire le coeur du peuple; elle use de l'eau sainte et de la doctrine mensongère du bonheur céleste pour passionner la multitude. Parmi toutes les mauvaises doctrines, il n'y en a pas qui causent aux bonneis mocurs de si déplorables détriments. Sous le règne de Minh-mang elle a été plusieurs fois sévèrement prohibée", etc., etc.

«En voici maintenant la partie dispositive.

10. "Les Chrétiens en place (fonctionnaires) dans la capitale auront un mois, et ceux des provinces trois mois pour abjurer; s'ils le font, ils seront conservés dans leurs dignités; dans le cas contraire, ils seront destitués et réduits à la condition de simples particuliers. Si après leur destitution, ils restent incorrigibles, ils seront punis sans miséricorde comme les derniers du peuple.»

«Aux soldats et aux particuliers six mois sont accordés pour se présenter devant leurs magistrats et fouler aux pieds la croix en leur présence. Ceux qui après ce temps n'auront pas abjuré ou qui continueraient à pratiquer la religion

1) A l'occasion des préparatifs de guerre civile au Tong-king dont il a été parlé plus haut, Mgr. Ketord, anssitôt qu'il eut connaissance de ces monvements, écrivit à tons les prêtres de sa mission une lettre qu'ils devaient communiquer aux Chrétiens pour leur défendre de prendre part à ces «remuements rérolutionnaires». 
chrétienne en secret, - quelle que soit leur science dans les lettres, - ne seront point admis aux examens publics pour la collation des grades littéraires et quelle que soit leur habileté dans les affaires, ils ne pourront jamais occuper aucun emploi dans leur commerce ou dans leur Canton."

$2^{\circ}$. "Ceux qui auront caché des prêtres étrangers seront arrêtés et livrés aux tribunaux, parmi eux ceux qui seront reconnus être les chefs et les principaux coupables, seront punis de mort."

“Les prêtres européens arrêtés auront la tête tranchée et exposée pendant huit jours au haut d'un poteau, pour l'instruction publique; si ce sont des prêtres annamites ou des européens gradués seulement en religion, ils seront simplement décapités sur le champ.»

"Ceux qui dénonceront secrètement ou feront arrêter un prêtre européen, recevront une récompense de 300 taels d'argent. Ceux qui dénonceront ou feront arrêter un Annamite ou un Européen simplement gradué, recevront une récompense de 100 taels, etc."

$3^{\circ}$. “Ordre est donné aux mandarins d'arrondissement et de sous-arrondissement de faire de fréquentes visites dans l'étendue du territoire de leur juridiction, pour rechercher les chrétiens, les instruire, leur apprendre ce qu'ils doivent éviter et ce qu'ils doivent admettre. Quant à leurs maisons de culte, les résidences de leu's prêtres, leurs provisions de vivres, grenier's de riz, leurs murs d'enceinte et leurs haies de bambous, - il faut les brûler, les détruire et les dissiper entièrement, combler leurs souterrains, abattre leurs vedettes, et défendre sévèrement aux chrétiens de l'un et de l'autre sexe, de se rassembler pour écouter les prédications de leur mauvaise religion. En cas de résistance, si les mandarins d'arrondissement et de sous-al'rondissement ne se sentent pas en force pour la réduire, ils en avertiront les grands mandarins de provinces qui viendront avec la force armée pour y mettre ordre, - sans se laisser intimider, mais aussi en agissant avec prudence, de manière à soumettre les coupables sans les exaspérer."

$4^{\circ}$. "Si après cela, des prêtres européens restent encore cachés parmi le peuple, et qu'ils soient découverts, les chefs de canton et les maires de communes où la découverte aura été faite seront punis suivant la loi portée contre ceux qui recèlent des criminels (c'est-à-dire de la même peine que le criminel lui-même), et le mandarin de sous-arrondissement sera puni suivant la loi portée contre les surveillants négligents, c’est-à-dire, qu'il recevra 100 coups de bâton et sera destituć. Les mandarins d'arrondissement ne cumulant pas les fonctions de chef de sous-arrondissement, perdront trois grades et recevront 90 coups de bâton; les mandarins de la justice civile et criminelle perdront deux grades et recevront 80 coups, les grands mandarins gouverneurs de province ne perdront qu'un grade et ne recevront que 70 coups de bâton."

«Pour avoir recelé un prêtre indigène, lorsque le fait sera prouvé, les mêmes fonctionnaires seront punis de peines graduées d'une manière analogue, 
mais proportionnellement moindres, - les chefs de cantons et maires de communes, au lieu d'être punis de mort, devant seulement recevoir 100 coups de bâton et perdre leurs places," - c'est ainsi, dit le décret, en terminant, que la rigueur sera unie à l'humanité et que la force sera tempérée par la clémence, le peuple se régénérera peu à peu, abandonnant le mal pour entrer dans les voies de la justice.»

La publication de cet édit, qui ne faisait en somme, que remettre en lumière et renouveler avec quelques rigueurs de plus, les dispositions des édits précédents restés toujours en vigueur, n'apporta pas du reste un changement très-marqué à la position de nos missionnaires et de leurs chrétiens; ce fut seulement l'aggravation d'un état de choses qui était déjà déplorable. Déjà lors des mouvements insurrectionnels qui íclatèrent au commencement de 1855, les bruits les plus alarmants avaient ité répandus; on disait que les auteurs et les chefs de cette róbellion étaient les missionnaires et les prêtres indigènes, et ce qui était plus alarmant encore, car ce n'était pas dénué de toute vraisemblance, on annonçait que les mandarins allaient venir avec leurs troupes pour cerner les villages chrétiens, brûler et piller ceux où ils trouveraient des prêtres et surtout des missionnaires, et en massacrer tous les habitants. Ces bruits avaient jeté l'effroi parmi les chrétientés, nos missionnaires avaient été obligés de redoubler de précautions pour se dérober aux recherches, à fuir pendant la nuit d'une retraite à une autre, ayant peine souvent à trouver un lieu où reposer leurs têtes.

«Depuis que le nouveau décret a été publié, disait Mgr. Retord, nos «prêtres annamites dans bien des endroits ne sont guère plus à leur aise que «nous. Les dix barres d'argent promises à celui qui en arrêtera un sont une «amorce trop attrayante pour ne pas tenter la cupidité des méchants et les «exciter à aller à leur recherche. De plus, un grand nombre d'espions, les uns «venus de la capitale, les autres envoyés par les mandarins locaux, traversent «le pays dans tous les sens et sous toutes les formes, et se glissent partout «jour et nuit. En outre dans chaque canton les mandarins ont établi des espèces «de commissaires de police qui, accompagnés de quelques hommes font la ronde «dans tout le canton, soit-disant pour découvrir les rebelles, mais dans le vuai «pour rechercher les prêtres, vexer les chrétiens et leur extorquer de l'argent.... «Dans bien des endroits les chrétiens, dans la crainte d'être vexés par les «païens des environs et les mandarins locaux, ont abattu eux-mêmes par pru«dence les églises et les maisons de Dieu, de sorte que les prêtres annamites «ont été obligés de renvoyer chez eux une partie de leurs catéchistes et de «leurs élèves, et eux-rnèmes sont forcés d'errer de maisons en maisons, de vil«lages en villages, sans trouver un lieu de repos."

«Or se figure aisément qu'au milieu de cette vie de proscrits sans cesse exposés à être livrés à des fonctionnaires corrompus, nos missionnaires sont souvent obligés d'avoir recours à l'argent pour acheter le secret de leur retraite 
et jouir d'un peu de paix. "Pour se mettre un peu à l'abri des coups de la «persécution, dit ' Mgr. Retord, il faut dépenser beaucoup d'argent, d'abord «auprès des grands mandarins de la province, puis auprès des petits mandarins «d'arrondissement et de sous-arrondissement, puis auprès des chefs de canton «et des maires de communes païns. Ce sont là comme autant de chiens affamés «à chacun desquels il faut jeter un os à ronger pour n'en être pas trop «fortement mordu. Si au moins, après avoir fait une certaine dépense, on avait «gagné la paix pour long tems, le mal serait supportable. Mais ces mandarins, «ces hommes en place, dont on se fait des amis avec de l'argent, sont bientôt «transférés ailleurs, ou destitués et emportés par la mort, et pour gagner les «bonnes grâces de leurs successeurs, il faudra faire de nouvelles dépenses, peut«être plus considérables que les premières.»

«Cependant, au milieu de ces circonstances critiques nos missionnaires furent assez heureux pour qu'aucun d'eux ne tombát entre les mains des autorités. Cela fut dû sans doute à toutes les précautions minutieuses dont ils surent s'entourer, à l'affection et à l'admirable dévouement de leurs chrétiens. Je crois qu'on peut l'attribuer aussi en partie à un fait que j'ai déjà eu l'occasion de faire observer (dans ma dépêche du 23 sept. 1852) et qui ressort également de plusieurs dispositions du dernier édit; c'est qu'en général les mandarins, ceux même d'un grade élevé, comme les Gouverneurs de province, sont peu portés d'eux-mêmes à la persécution. On en verra une nouvelle preuve dans l'incident que je vais rapporter. Je disais qu'aucun de nos missionnaires ne fut découvert et arrêté dans ces circonstances; il s'en fallut de peu cependant. Je laisse raconter Mgr. Retord:

«Le 8 Février dernier, les deux grands mandarins de la justice criminelle «et de la justice civile et le mandarin civil de l'arrondissement vinrent avec "deux éléphants, deux pièces de canon et 1500 hommes de troupes, bloquer «notre maison de Kenou, soi-disant pour s'emparer d'un chef de rebelles nommé «le Grand tigre, mais en réalité pour prendre des inissionnaires qu'ils croyaient «trouver là réunis au nombre de trois ou quatre. C'est le même individu qui «avait dénoncé et fait prendre Mr. Bonnard, qui dénonça notre maison de «Kenou comme un repaire de guerriers et une habitation de prêtres européens. «Vous savez que Kenou est en importance le second de nos établissements»... Mgr. Retorda explique comment Mgr. Jeantet qui était à la tête de cet établissement prévenu par des avis officieux de la visite qui se préparait, en profita pour mettre en sureté dans des cachettes ses effets les plus précieux, puis aller se cacher lui-même dans la maison du chef du canton sous le nom duquel le collége de Kenou était placé. Ce chef de canton qui habite dans un village voisin faisait preuve d'un grand dévouement en recevant Mgr. Jeantet chez Jui dans un moment aussi critique, car si l'évèque avait été pris dans sa maison, il aurait dû d'après la loi subir la même peine que ce prélat. „Cependant, «continue Mgr. Retord, deux jours se passèrent sans que les mandarins parussent 
"et Sa Grandeur se préparait déjà à rentrer chez elle, lors qu'un de nos élèves «vint avertir que deux païens du chef-lieu d'ar'rondissement avaient donné la «nouvelle que les grands mandarins prenaient des dispositions pour cerner le «village de Kenou avec la force armée cette nuit là même et effectivement le «lendemain matin tout le quartier du village où est situé notre collège était «cerné. La plus grande partie de nos élèves avaient eu le temps de prendre la «fuite, quelques-uns, des plus courageux qui étaient restés pour garder un peu «la maison, furent obligés de s'échapper comme ils purent parce que le dénon«ciateur les indiquait aux mandarins pour les faire arrêter. Notre maison resta “donc à la merci des soldats, qui mangèrent, pillèrent, brisèrent tout ce qui «leur tomba sous la main. Ce qu'ils ne pouvaient pas emporter ils le vendirent «aux paiens des environs, ils fouillèrent partout pour tronver les immenses «richesses qu'ils s'imaginaient être cachées dans cette maison; ils bouleversèrent "la boue des étangs, creusèrent et sondèrent le terrain dans l'intérieur de la «maison, dans la cour, dans les jardins, ils ouvrirent même des tombes encore «fraiches qu'ils croyaient renfermer des objets précieux»....

"Mgr. Retord énumère ici les divers vols, gaspillages et dégâts commis par «les soldats, dont il évalue le dommage à 2550 ligatures (ou $2500 \mathrm{fr}$.) ; puis il «ajoute: «Est-ce tout? non certes; outre cela, les soldats arrêtèrent un prêtre, «curé de la paroisse, un diacre, le chef de canton Cai-cam, et 15 autres per"sonnes, théologiens, élèves ou domestiques de notre maison, que le mandarin "renvoya le lendemain, ne gardant que le prêtre, le diacre ot le chef de canton «qui furent mis à la cangue et jetés en prison. Voilà donc encore une grande «affaire qui paraissait devoir durer longtemps; néanmoins au bout de cinq jours «tout fut terminé, le grand mandarin de la province donna ordre de délivrer “les prisonniers et de les renvoyer en paix chez eux, parce que ce n'était pas "cette espèce de gens que l'on cherchait, mais seulement le Grand Tigre chef «des rebelles, ou au moins, des prêtres européens. Le prêtre annamite ne fut «pas même interrogé sur sa qualité de prêtre et on ne proposa à aucun des "prisonniers de fouler au pied la croix, excepté au chef du canton, au moment «du blocus. Mais il leur répondit avec fermeté: «Messieurs les Mandarins, mes «ancêtres étaient chrétiens, et je le suis aussi, je veux l'être jusqu'a la mort. «Si dans cette maison vous trouvez des rebelles, des armes, ou même des "prêtres eurnpéens, je consens à être puni de la peine capitale. Sinon je demande "que celui qui nous a calomnieusement accusés soit puni de la même peine. "Les mandarins voyant sa fermeté ne lui parlèrent plus d'apostasier ni de fouler «la croix aux pieds. En renvoyant les prisonniers, ils rendirent aussi notre "horloge ainsi que plusieur's autres objets qu'ils avaient saisis; mais combien «en a-t-il coûté pour obtenir ce résultat? Simplement la bagatelle de $\mathbf{5 0}$ barres «d'argent ${ }^{1}$ ) et 300 ligatures de monnaie plus 425 ligatures dépensées par les

1) Une barre d'argent vaut environ 100 francs. 
«prisonniers ou par les hommes d'affaires. Une barre d'argent vaut maintenant «de 85 à 90 ligatures. Si donc maintenant vous calculez et ajoutez toutes ces «sommes, vous trouverez que cette affaire du blocus de Kenou nous a déjà кcausé un dommage de 7775 ligatures, c'est-à-dire de 4.665 .000 sapèques «(environ 9000 francs). Et ce n'est pas encore tout: ce blocus a en lieu avant "la publication du fameux édit cité plus baut; après la publication de cet Edit, «il fallut encore donner cinq barres d'argent au petit mandarin du sous-arron«dissement; il faudra probablement abattre une partie de la maison pour npouvoir conserver l'autre, et nos élèves sont toujours en grande partie dis“persés de côté et d'autre, sans savoir quand ils pourront être réunis.... et «Mgr. Jeantet est toujours en fuite sans espérance prochaine de rentrer dans «son séminaire et reprendre ses fonctions, etc "

"J'ai cru devoir m'étendre un peu sur ces citations, M. le Comte, parce qu'elles offrent une peinture saisissante et sans aucun doute exacte de la position de nos missionnaires au milieu de ce pays travaillé par l'esprit de révolte, en face de ce pouvoir ombrageux qui tient toujours une menace de mort suspendue sur leurs têtes, et qu'elles peuvent ainsi donner une juste idée de l'aspect que présentaient les aflaires en Cochinchine à une époque assez rapprochée de celle où le Plénjpotentiaire de S. M. Impériale devait y arriver pour négocier un traité. Si l'on ajoute ces circonstances à celles que j'ai déjà exposées précédemment, si l'on considère surtout quelle avait été depuis plus de trente ans la politique du Gouvernement Annamite, encore à ce moment représentée par le vieux Ministre qui avait été pendant de longues années le plus ferme conseiller du roi Minh-mang dont le nom est resté grand dans la mémoire de ces peuples, par la bonne administration qu'il sut donner à ses états, et par la rigueur implacable avec laquelle il poursuivit Ja religion chrétienne, on comprend facilement que, dans l'entreprise que nous tentions, celle d'obtenir d'un seul coup de ce gouvernement un traité qui n'était rien moins que le renversement de toute sa politique à l'égard des barbares, c'est-à-dire des Européens, ce n'était pas sur ses dispositions favorables que nous devions compter.

"Cependant, même avec ces désavantages et ọn ne pouvant au fond espèrer le succès que de la crainte qu'une démonstration subite et énergique pourrait inspirer à la Cour de Hué, il n'était peut-être pas impossible, si l'affaire avait été conduite avec promptitude, décision et habileté, d'amener le Gouvernement Annamite à accéder au moins à une partie de nos demandes et à conclure un traité. Il aurait fallu pour cela que M. de Montigny, au lieu d'envoyer à l'avance un des bâtiments dont il disposait à Tourane avec une notification où il annonçait l'objet de sa mission dans des termes aussi impérieux dans le fond, que pompeux dans la forme, notification qui avait le double inconvénient de prévenir le Gouvernement Annamite, de lui donner le temps de la réflexion, et d'irriter son orgueil par une nouvelle menace non suivie d'un effet immédiat, 
se rendit lui-même sans perte de temps à Tourane avec les trois bâtiments qui l'avaient accompagné dans sa mission à Siam, et se présentât tout d'abord à la Cour de Hué avec un langage pacifique mais ferme, et l'appui de la force derrière lui, Les évènements ne tardèrent pas à le démontrer.

"Le Catinal arriva vers le 15 sept. sur la rade de Tourane et se dirigea dés le lendemain ver's l'entrée de la rivière qui conduit à la capitale, où l'officier chargé de remettre les dépéches de $M$. de Montigny fint reçu poliment et contraignit en quelque sorte le mandarin commandant le fort le plus rapproché à les recevoir. Mais deux ou trois jours après, la notification fut rapportée au Commandant du Catinat qui avait regagné le mouillage de Tourane par des employés «du plus bas étage»; ils ćtaient en outre chargés de lui faire connaître que les lois du royaume s'opposaient à ce que des ćtrangers descendissent à terre sous quelque prétexte que ce fut. Le Commandant du Catinat se montra justement offensé du refus du Gouvernement annamite de recevoir les communications du représentant de la France et d'un pareil message; il y vit une insulte, ce que c'était en effet, et se décida à adresser aux autorités de Tourane un ultimatum par lequel il exigeait que le Gouvernement annamite ordonnât immédiatement le désarmement des for'ts de la côte, qu'il répondit officiellement à la notification du Plénipotentiaire de France, et que l'espèce de quarantaine imposée au Catinat contre les usages du monde civilisé fut levée. Un délai de six jours était accordé aux autorités de Tourane pour faire droit à ces demandes. On sait quel fut le résultat de ces premières difficultés. Je ferai seulement remarquer en passant que l'opinion que j'exprimais il y a cinq ans, «que «les forts qui défendent le port de Tourane seraient au besoin facilement «enlevés" (dépêche du 23 sept. 1852) se trouva pleinement justifiéé par l'acte de virueur du Commandant Le Lieur.

«Cependant quel avait été l'effet produit à la Cour de Hué par la nouvelle de l'arrivée d'un navile de guerre français à Tourane apportant des dépéches d'un Envoyé dont il devait etre prochainement suivi? Voici à ce sujet quelques détails puisés dans les informations qui mont été données par des personnes qui ont pris part à la mission de Cochinchine et ont été à même de savoir exactement comment les choses se sont passées." Le roi Tu Duc, en apprenant ces nouvelles était entré dans une violente colère, avait déclaré traître envers l'Etat et punissable de mort tout Annamite qui s'approcherait du navire ou des barbares qui descendraient à terre. En vain son Ministère tout entier réuni en conseil remit au roi un rapport dans lequel il le suppliait d'accepter la lettre officielle de l'Envoyé de France et de conclure un traité d'amitié avec cet Empire. Le vieux Long-koué avait retrouvé l'énergie de ses jeunes années; connaissait-il le contenu des dépêches laissées entre les mains du Commandant du fort? C'est ce qu'on ne pourrait affirmer. Toujours est-il qu'il s'éleva avec violence contre les conclusions du rapport ministériel, déclara que le Royaume devait poliment mais péremptoirement refuser d'entrer en relations avec des 
gens qui s'annonçaient de cette manière, et qu'il était en outre d'une nécessité urgente d'appliquer dans toute la rigueur l'Edit lancé vingt mois auparavant contre les Chrétiens, cause première de tout ce qui arrivait. Ce fut alors que les Communautés de Mgr. Pelderin, vicaire apostolique de la Cochinchine septentrionale, son collége et autres établissements furent détruits, et ses principaux chrétiens emprisonnés; que lui-même s'échappant des environs de Huéà travers mille dangers, fut obligé de fuir vers la côte, et après avoir erré pendant 5 on 6 jours dans des montagnes sauvages, parvint ì se réfugier à bord de la Capricieuse qui était arrivée sur les entrefaites à Tourane. Un grand Mandarin, exemple unique d'un Chrétien dans ce rang élevé qui depuis plusieurs années et même depuis la promulgation du dernier édit de persécution, occupait de hautes fonctions dans la capitale, bien que le roi et ses ministres connussent quelle était sa religion, fut destitué de toutes ses charges et dignités et mis à la chaine.

"Ces premières mesures de réaction et de vengeance étaient à peine exécutées quand la nouvelle du combat de Tourane arriva à Hué; le gouvernement annamite se montra aussi abattu à cette nouvelle qu'il avait paru énergique et résolu quelques jours auparavant. Effrayé de ce que le Calinat avait pu faire à lui seul, il se demanda de quoi il n'était pas menacé à l'arrivée des autres navires français, s'il persévérait dans la voie imprudente où il s'était engagé. Le parti de la paix l'emporta à ce moment dans l'esprit du roi, et la corvette la Capricieuse étant arrivée peu de jours après à Tourane, le Gouvernement annamite transmit à $M$. le Commandant Collier une dépêche pleine de dignité et de modération, dans laquelle les bases indiquées dans la notification de $\mathbf{M}$. de Montigny étaient acceptées et il était dit qu'on attendait avec impatience l'arrivée du Plénipotentiaire français pour les convertir en traité; - les navires français seraient approvisionnés pendant tout le temps qu'ils resteraient à Tourane, et les Français pouvaient circuler en liberté dans toute l'étendue de la presqu'île.

“On ne pent croire sans doute, que ces nouvelles dispositions étaient bien sincères, mais il n'est pas douteux, d'après ces données, que si notre Plénipotentiaire était arrivé à cette époque, comme il aurait pu et dû le faire, en s'embarquant sur le Catinat ou la Capricieuse au lieu de s'attarder ì Bangkok avec un faible bâtiment qui ne ponvait pas tenir la mer contre la mousson, il eut obtenu sinon tout, du moins la partie la plus importante de ce qu'il avait à demander au Gouvernement Annamite, et accompli l'objet de sa mission, en concluant un traité qui aurait ouvert de nouveaux débouchés à notre commerce et assuré à nos missionnaires d'inappréciables garanties. On comprend trop bien qu'un délai de près de quatre mois venant s'interposer entre les opérations du Catinat et de la Capricieuse et l'arrivée de M. de Montigny devait ôter toutes chances de réussite ì une entreprise qui s'appuyait déjà sur des moyens insuffisants, auxquels on ne pouvait suppléer que par beaucoup de promptitude, de tact et d'énergie. 
"Cette trop longue relâche, dit une des personnes auxquelles je dois mes informations," usa peu à peu notre prestige aux yeux de la population, en «contact permanent avec nous; le peuple et les autorités s'accoutumèrent à nous «voir sans crainte et à nous redouter chaque jour un peu moins. En vain la «rare prudence et la parfaite rectitude de jugement de $M$. le Commandant "Collier surent maintenir les choses sur" un pied de convenable neutralité qui «allait tous les jours en s'atténuant. Déja l'on n'attribuait plus la prise des «forts de Tourane qu’à la lâcheté de leurs défenseur's et à la faiblesse de «leurs garnisons; de nouvelles troupes arrivaient sans cesse à Tourane, troupes «d'élite sur lesquelles le Gouvernement comptait et qu'il n'eut pas été fảché «de voir se mesurer avec nous. Les édits contre les Chrétiens étaient maintenus, "et les prisons ne semblaient plus devoir se vider."

"Ce fut à ce moment que M. de Montigny arriva à Tourane sur le Marceau. Mais le moment favorable était passé, et dans l'opinion d'un témoin de ces évènements, «la partie était perdue définitivement depuis deux mois au «moins." Notre Plénipotentiaire essaya d'entrer en négociations, mais elles vinrent se briser contre «le calme poli et la résolution inflexible» des mandarins qui représentaient le roi de la Cochinchine en cette occasion. Ne pouvant obtenir satisfaction sur aucun point, Montigny voulut essayer de terrifier encore une fois le Gouvernement annamite, et le 31 janvier all matin il notifia aux autorités de Tourane l'ultimatum dont j'ai l'honneur de joindre ici copie, ultimatum dans lequel il leur accordait un délai de 24 heures pour se décider à entrer en négociation formelle et régulière avec lui. M. le Commandant Collier ne crut pas devoir, dans ces circonstances, appuyer à la lettre les menaces du Plénipotentiaire faites d'ailleurs à ce qu'il parait sans son aveu, et je crois que cette résolution, au point où les choses en étaient arrivées, était sage et judicieuse. Il fallut donc retirer l'ultimatum si imprudenment notifié le matin et ce fut Mgr. Pellerin qui fut chargé de cette mission délicate. L'évêque se rendit à Tourane, déclara que le Plénipotentiaire de France voulait bien encore, pour donner une nouvelle preuve de ses intentions pacifiques retirer son ultimatum, si le Gouvernement annamite consentait au moins à conclure une convention avec la France. L'envoyé du roi d'Annam, comprenant plus que jamais tous les avantages de la politique pleine de prudence et de calme qu'il avait suivie jusqu' alors “remit en souriant l'ultimatum à $\mathrm{Mgr}$. Pellerin» et fixa le 2 Février pour le jour de la première réunion.

«Nous étions bien loin alor's du combat de Tourane», dit l'un de mes informants, «depuis cette époque nous avions perdu un à un tous nos avantages. «Le rusé diplomate qui nous était opposé avait parfaitement jugé la situation «et paraissait moins disposé que jamais à nous accorder quoique ce fût. Aussi «ne se montra-t-il jamais plus difficile et plus hautain que dans cette mémo«rable conférence du 2 février; jamais non plus, il faut l'avouer plus de politesse «ne vint couvrir plus de raideur." 
«Toutes les demandes de notre Plénipotentiaire rejetées une à une, toutes les concessions refusées, et cela lorsque le Commandant de nos forces navales s'était refusé à tout nouvel acte d'hostilité, rendaient nécessaire une séparation immédiate. Mais il fallait restituer nos conquêtes avant de quitter Tourane, et ce fut là l'objet de la dernière notification remise le 6 février à l'envoyé extraordinaire du roi de la Cochinchine. Je crois inutile de joindre ici la copie de ce document, qui me fut communiqué, à mon passage à Hongkong, ainsi que le Prọjet de traité, par M. de Montigny lni-même. S'il m'est permis d'exprimer mon opinion sur ce dernier acte de la mission de cet agent, je dirai que la première partie de cette note était conçue en termes dignes et convenables. Malheureusement derrière le Commandant de nos forces navales adésirant «éviter tout acte d'hostilité inutile avant la ríception de nouveaux ordres de «son Gouvernement," et restituant généreusement le matériel des forts qu’il avait pris, apparaissait le Plénipotentiaire éconduit et irrité, menaçant le Gouvernement annamite des mesures les plus énergiques «et de représailles", et cela à propos de persécution religieuse et de nouvelles exécutions pour le fait de pratiquer la religion chrétienne. Si M. de Montigny avait inieux connu ou avait songé à la haine implacable que le Gouvernement annamite nourrit contre la religion du Christ et contre nos intrépides missionnaires, il eut, au moment de quitter ces rivages où nous venions de nous présenter encore une fois sans avoir pu rien effectuer que l'inutile capture de quelques forts, gardé un silence absolu sur sa mission religieuse (et cela s'applique également à la communication du 'Traité dont il a remis une copie en partant aux autorités annamites). Parler de persécution religieuse et nouvelles exécutions, c'était trop découvrir à ce Gouvernement quel était le point sensible, l'endroit vulnérable, c'était en quelque sorte lui révéler par quelles gens nous avions été appelés, et lui désigner trop clairement ceux sur qui il devait faire tomber sa vengeance.

«Nous quittâmes Tourane», dit l'observateur que j’ai déjà cité, "sur ces «entrefaites raillés et méprisés, nous y étions pour ainsi dire arrivés en vain«queurs, nous en sortions en vaincus. A notre arrivée dans ce pays nous avions «trouvé la persécution diminuant, et nous l'avons laissće plus vivace que jamais. «La prise des forts de Chun-tsa en 1856, comme le bombardement de 1847 «avaient inspiré une salutaire terreur de nos armes, un juste respect du nom «Français au Gouvernement annamite, et nous avons laissé derrière nous une «idée plus que médiocre de la puissance et de l'intelligence françaises"...

"S'il est quelque chose qui doive nous consoler, M. le Comte, du triste résultat qu'a eu la mission de Montigny, c'est de penser, - et telle est ma conviction, que le traité que cet envoyé aurait pu conclure, serait resté sans exécution par l'absence d'une garantie indispensable qui lui aurait manqué dans les circonstances oì l'on se trouvait, je veux dire la permance d'un bâtiment de guerre français sur les lieux, suivie, dans un bref délai, de l'occupation d'un 
point du territoire annamite à proximité de la capitale. Une autre circonstance, heureuse sous le rapport de la facilité de réparer l'échec que nous avons éprouvé en Cochinchine, c'est la présence das forces considérables que le gouvernement de S. M. Impériale possède, en ce :moment, dans ces parages et qui, suivant la tournure que prendront les affaires de la Chine, pourraient être employées à une expédition devant laquelle la Cour de Ilué ne pourrait qu'abandonner toute idée de résistance. J'ajoute à ce sujet qu'ì moins que, dans le courant de l'hiver prochain, on ne se trouve en mesure de reprendre les hostilités contre Canton et d'en finir avec ce grave embarras, des opérations sérieuses en Chine semblent ajournées au moins jusqu'au printemps prochain, et qu'ainsi, si le Gouvernement de l'Empereur s'y décide, une expédition en Cochinchine pourrait avoir lieu avant cette époque vers le commencement de janvier, par exemple, saison la plus favorable pour opérer dans ces parages.

"Il me reste à indiquer à V. E., quels seraient, dans mon opinion, les objets qu'on devrait se proposer et la manière dont il conviendrait de procéder en dirigeant une nouvelle expédition vers la Cochinchine pour y reprendre les clıoses au point où les a laissées le départ de M. de Montigny. A cet égard, M. le Comte, V. E. me permettra encor'e une fois de me référer à la dépêche que j'ai eu l'honneur d'adresser à son Département à la date du 23 sept. 1852.

«Les bases de traité et le mode d'action, que je proposai à cette époque me paraissent encore, sauf quelques modifications dont je vais signaler les principales, ceux qui conviendraient le mieux dans les circonstances actuelles. Dans ma dépéche du sept. 1852, j’indique, parmi les demandes ì faire au gollvernement annamite comme garanties nécessaires à l'exécution du traité, etc. - , la cession du port et du territoire de Tourane ou d'un antre point offrant une position militaire. Je pense maintenant que c'est Tourane même et la presqu'île de ce nom, et non aucun autre point qu'il faudrait occuper', par la raison déterminante de la proximití de cette position de la capitale et de l'action beaucoup plus puissante qu'elle nous assurerait sur la Cour de Hué. Je crois d'ailleurs aussi qu'elle serait la plus avantageuse sous le rapport commercial. Je disais dans la même dépîche, que «dans le cas où le point cédé «serait le port de Tourane, cette cession pourrait donner lieu ì une indemnité «à payer par la France." Mon opinion aujourd'hui n'est plus la míme à cet égard. Je pense que Tourane, sans grande valeur comme territoire, devrait nous ćtre cédé purement et simplement comme garantie de l'exécution du traité et de meilleurs procédés à l'avenir. Je suis d'avis que nous devrions, en outre, exiger du Gouvernement cochinchinois une indemnité pécuniaire. $1^{\circ}$. à titre de restitution (si le fait est avéré) pour une somme de 270 mille piastres, (environ 1800 mille francs), qu'on m'assure avoir été payée par la France sans aucune compensation en exécution du traité de 1787. $2^{\circ}$. comme réparation poul la mise à mort de missionnaires français, au moins pendant le règne actuel, et les nombreuses spoliations dont nos missionnaires ont été victimes 
par suite des édits de persécution; $3^{\circ}$. à titre d'indemnité de guerre, pour l'insulte faite à la France en refusant de traiter avec son envoyé et la cońteuse expédition à laquelle ce refus aura donné lieu.

«Le projet de traité présenté par M. de Montigny comprenant d'ailleurs toutes les demandes que nous avons à faire au Gouvernement annamite, pourrait servir de base à une nouvelle négociation, sauf quelques changements à introdıile dans l'ordre des stipulations, qui ne me paraissent pas classées suivant leur importance, ainsi que dans la rédaction des divers articles. Les garanties à donner à la sécurité future de nos missionnaires, à leur inviolabilité comme sujets français, devraient ì mon sens, figurer en première ligne.

«Je n'ajouterai que quelques mots, M. le Comte, en ce qui concerne le mode d'exécution. Si V. E. entre dans les vues que j'ai l'honneur de lui soumettre, et que le Gouvernement de l'Empereur voulût profiter des forces imposantes dont il dispose en ce moment dans ces mers pour prendre en Cochinchine une position qui nous offrirait, à la fois réparation pour le passé et des avantages considérables pour l'avenir, Elle pensera sans doute comme moi, que tout en conservant à une nouvelle démonstration vis-à-vis de la Cour de IIué un caractère diplomatique et courtois, à moins que, d'ici là, de nouveaux outrages de sa part ne viennent aggraver l'état des choses, il conviendrait de procéder dans cette entreprise sans tergiversation et sans crainte d'employer la force pour obliger ce Gouvernement à souscrire à notre juste demande. Je suis convaincu que plus nous nous montrerons décidés, moins on songera à nous résister. Aussi, comme je suis d'avis que, dès l'arrivée de l'expédition à Tourane, en même temps que le Plénipotentiaire de $\mathrm{S}$. M. Impériale entrerait en négociation avec le Gouvernement annamite, nous y prissions tout d'abord pied en occupant les forts, etc. Si cette première démonstration ne suffit pas, il faudrait également se rendre maitre de ceux qui défendent l'entrée de la rivière de Hué et menacer cette capitale elle-même. Mais d’après ce que l'on sait de la faiblesse, sous le rappor't militaire, de ce Gouvernement de l'Extrême $\Lambda$ sie dont l'orgueilleuse arrogance prend sa source dans la distance et l'indifférence de l'Europe, d'après les renseignements mêmes qui ressortent de la dernière malencontreuse mission, je crois très probable que le monarque annamite attend jusque là pour se soumettre à nos conditions.

Veuillez, etc.

(sig.) $\Lambda$. Bourboulon.

Le décret suivant rédigé quatre mois après le départ de $M$. de Montigny de Tourane ne pourait laisser aucun doute sur les sentiments du gouvernement annamite; nous le reproduisons malgé sa longueur à cause de son importance. M. KLeCzkowski se l'était procuré lors de la mission dont il fut chargé et dont nous parlerons plus loin. 
Déret annamite 27 mai 1857.

Traduction un peu libre et abrégée, mais fidèlement quant au sens, d'un décret composé par les Mandarius de Phâ-xuan, présenté au roi annamite $\mathrm{Ta}$ Đuc, le 3 de la $5^{\mathbf{e}}$ lune de cette anuée, c'est-àdire le 25 mai 1857 , et que Sa Majesté a dit-on approuvé et beaucoup loué.

Grand Saint, examine ce qui suit. Par votre brillante doctrine, vos études saintes et profondes et la rectitude de votre coeur, vous observez dans toute leur perfection les huit principes de la conduite humaine "). Par le bon système de votre gouvernement, la paix règne dans l'Empire; vous pratiquez dans toute leur intégrité les neuf règles d'une bonne administration $\left.{ }^{2}\right)$. Vous protégez les peuples tributaires et vous êtes plein de bonté envers les étrangers. A l'intérieur comme à l'extérieur, vous n'omettez rien de ce qui pent rendre le peuple heureux. Ceux qui sont près de vous sont dans la joie, et ceux qui sont éloignés cherchent les moyens de vous approcher. Vos peuples jouissent de la paix et les Barbares du dehors vous sont soumis. Néanmoins, si nous devons nous féliciter de la tranquillité présente, nous ne devons pas pour cela être sans inquiétude pour l'avenir. Les anciens n'ont jamais été tristes et inquiets même dans les moments de loisir et de paix [?].

Maintenant nous voyons les Chrétiens annamites qui ne se soumettent qu'en apparence à vos ordres, sans abandonner véritablement leur fausse religion. Nous voyons les Cambodgiens qui se tournent vers la doctrine des Européens, de sorte que leur soumission à votre autorité devient bien problématique. Les Barbares d'Europe viennent de leur lointain pays vous faire des demandes dont ils pourront bien $\grave{a}$ la fin ne pas supporter tranquillement le refus. Ces trois ennemis peuvent se réunir dans une commune action, pour faire réussir leurs projets. Nous avons donc de graves sujets d'inquiétudes malgré notre prospérité. Sachons donc calculer juste. N'oublions point de nous munir des chiffons nécessaires pour boucher, au besoin, les fissures de notre barque; ne méprisons point les piqures des abeilles qui, négligées, pourraient devenir de graves ulcères....

Voici done ce que nous proposons; pour abolir la religion chrétienne, et propager la bonne doctrine, il faut employer envers les Chrétiens un redouble-

1) Ces huit principes sont: connaître les choses, acquérir un savoir profond, affermir son intention, rectifier son cœur, corriger ses défauts corporels, régler sa maison, gouverner le royaume, faire régner la paix partout. (Note du Traducteur).

2) Ces neuf règles sont: se corriger soi-même, honorer les sages, aimer ses proçhes, respecter les grands vassaux, connaître bien les magistrats, être le père du peuple, faroriser les artistes, âtre doux envers les étrangers, et protéger les royaumes tributaires. (N.T.) 
ment de sévérité pour leur faire abjurer leurs erreurs; et récompenser largement le mérite de ceux qui contrịbneront à arrêter les prédicateurs de cette doctrine dépravée. Pour nous prémunir contre les attaques des Cambodgiens, il faut garder avec soin nos frontières, en les garnissant de forts qui en ferment l'entrée. Pour nous préserver contre les entreprises des Barbares européens, il faut multiplier sur nos côtes, les difficultés et les périls afin de leur ôter la possibilité de les aborder.

En unissant la clémence et la rigueur, la vertu et la sévérité dans un sage tempérament, nous pourrons être sans indulgence envers les Clrétiens opiniâtres, et sans crainte au sujjet des barbares étrangers. Le Gouvernement de Votre Majesté à l'intérieur comme à l'extérieur étant établi sur de tels principes sera la source d'un règne long et paisible, et fournira des moyens efficaces pour opérer une grande amélioration dans les moeurs... Nous disons d'abord quil faut propager la bonne doctrine avez zèle, user d'une grande sévérité envers la mauvaise religion de Jésus, et récompenser largement le mérite de ceux qui aideront ì la doctrine en arrêtant ses prédicateurs. D'après l'édit donné la $7 \mathrm{e}$ année de votre règne, les prêtres européens qui seront arrêtés, auront la tête tranchće sur le champ; leur tête restera exposée trois jours en haut d'un poteau, puis jetée avec leur corps dans les eaux de la mer ou des fleuves. Les disciples des prêtres européens et les prêtres annamites doivent aussi être décapités sur le champ. Pour apaiser une rébellion, il faut tîcher de se saisir des chers, comme pour détruire une horde de scélérats, il faut d'abord mettre à mort les principaux coupables. Or les prêtres sont les principaux coupables et les chefs des Chrétiens; il y a des lois pour les punir cornme ils le méritent; mais dans les provinces elles sont bien mal exécutées, de sorte que beaucoup de ces coupables échappent à leur rigueur. Même les prêtres européens entrent dans le Royaume et en sortent à toute époque sans qu'on suive leurs traces pour les arrêter. Il y a beaucoup de prêtres annamites, mais bien peu ont été mis entre les mains de la justice. En serait-il ainsi si tous les mandarins des Provinces faisaient cordialement leur devoir? Il faut donc leur ordonner de redoubler de soin, pour prendre des informations sur tous les villages où pourraient se trouver des Prêtres. Les chefs de cantons et les maires de villages qui les arrêteront dans leurs territoires, ne seront pas punis pour les avoir laissé pénétrer chez eux. S'ils échappent par la fuite, ils devront les poursuivre et pourront les arrêter partout où ils les rencontreront. Ceux qui seront connus pour les avoir cachés, ou avoir favorisé leur fuite sernnt punis selon toute la rigueur des lois De cette manière, les lois obtiendront leur effet, et n'auront pas été portées en vain. Dans l'édit susmentionné il est encore statué que les disciples des prêtres annamites seront marqués aux joues et condamnés à l'exil dans les postes militaires établis loin de la mer. Par cette mesure, Votre Majesté manifeste la bonté de son coeur et la clémence de son Gouvernement. Elle inflige des peines légères, afin de ramener les coupables par la 29 
douceur... Mais les Chrétiens qui depuis si longternps ont été tant de fois éclairés par les saintes instructions de Votre Majesté et si souvent avertis par les prescriptions de la loi et qui cependant, n'ont point abandonné leur mauvaise religion, ne doivent plus jouir du bénéfice d'un système de douceur qu'ils méprisent avec tant d'ingratitude. Puisqu'ils sont indociles, il faut vaincre leur opiniatreté par la sévérité des châtiments. Sans cela, il est à craindre que jamais leur entêtement ne soit brisé. Il est vrai, ils sont trop nombreux pour qu'il convienne de les mettre tous à mort, mais au moins pourquoi laisserait-on vivre ceux qui, dénoncés, arrêtés et livrés aus mandarins s'obstinent dans leur aveuglement et refusent opiniâtrement d'abandonner leur mauvaise religion. D'ailleurs, aujourd'hui, ce sont de simples disciples des Prêtres; qui sait si demain, ils ne seront pas faits prêtres? Si on leur pardonne, ne feront-ils pas comme plusieurs, ne sortiront-ils pas du Royaume pour chercher à établir des intelligences avec les étrangers? Les envoyer en exil, c'est encore un grand danger, car souvent ils prêchent leur. Religion au lieu de leur exil et lui attirent de nombreux partisans. Ce sont des choses qu'il ne convient pas de laisser subsister. Ainsi donc les Chrétiens disciples des prêtres annamites, qui seront découverts et arrêtés, s'il sont endurcis dans leurs erreurs, seront condamnés à la strangulation. On leur accordera néanmoins quelque temps de réflexion; puis on les instruira et examinera de nouveau. S̈ils sont disposés à clranger de conduite, s'ils abjurent leur mauvaise doctrine, et promettent ainsi que leurs femmes et leurs enfants de suivre les bonnes habitudes du pays, en faisant des sacrifices aux ancêtres aux époques déterminées, sans jamais plus observer les prescriptions de la religion de Jésus, ce sera là une marque d'un repentir sincère de leur ancien crime dont on leur accordera le pardon en les mettant en liberté. Mais s'ils restent endurcis dans leur's erreurs préférant plutôt mourir que de les abandonner, ou si après les avoir rejetées, ils y retombent de nouveau, ne les reniant à l'extérieur que pour les suivre en cachette, ils seront impitoyablement exécutés; une conduite si coupable ne peut pas rester impunie; il s'agit de détruire les mauraises moeurs, et de défendre la bonne doctrine; il faut pour cela agir avec sévérité.

Mais pour instruire les ignorants et les soustraire au supplice, il faut agir avec méthode. Les Chrétiens croient une foule d'absurdités sur le Maître du Ciel, le Ciel, l'Enfer, et l'Eau sainte, etc., que leurs Prêtres ont mises sous forme de prières qu'ils leur font chanter le matin, et réciter le soir; d'où il arrive qu'ils s'en imprègnent au point de ne plus rien comprendre autre chose. Hé bien! imitons leurs Prêtres en cela, pour ouvrir l'intelligence des Chrétiens et leur enseigner la bonne Doctrine, et insensiblement nous les verrons, de jour en jour, éviter le mal et pratiquer le bien. Nous avons dans les dix articles publiés jadis sous le règne de Minh-mang, une doctrine qui ne laisse rien à désirer pour former de bons citoyens; faisons la réciter aux Chrétiens en place de leurs absurdes priĉres; obligeons tous ceux d'entre eux qui ont des enfants aux 
écoles de leur donner ces dix articles comme livre classique qu'ils réciteront assidúment pour former leur coeur à la vertu et qu'ils étudieront avec soin pour apprendre les caractères chinois. Mais comme ceux qui étudient ou savent les lettres chinoises sont peu nombreux, et que ceux qui ne les savent pas pourront difficilement comprendre, en langue chinoise, les sublimes instructions de ces dix articles, c'est pourquoi il faudra les traduire en langage annamite sous forme de chant, pour mieux les inculquer dans l'esprit du peuple. On en imprimera un grand nombre d'exemplaires que l'on répandra dans les villages où il $\mathrm{y}$ a des Chrétiens. Les Maires les liront et les expliqueront une ou deux fois au peuple pour qu'ensuite il les apprenne de mémoire et les récite chaque jour. Par là, le peuple apprendra la bonne doctrine, s'en pénétrera de plus en plus, connaitra les vertus qu'il faut pratiquer ainsi que les erreurs et les mauvaises habitudes dont il doit se corriger.

Dans l'Edit de la $7^{\text {A }}$ année de Votre Règne une récompense de 300 taels est promise à ceux qui arrêteront un prêtre européen; ceux qui arrêteront un prêtre annamite, ou le disciple d'un prêtre européen, recevront une rénumération de 100 taels d'argent. En cela, Votre Majesté a eu intention de récompenser le mérite. Mais ceux qui font des actions méritoires n'agissent pas tous par des vues d'intérét. Le plus grand nombre au contraire est mû par le désir de la gloire. Ceux dont l'intérêt est le mobile sont beaucoup moins empressés et moins ardents que ceux que la gloire pousse. Il faut dans une affaire si importante, mettre en jeu ces deux mobiles. Ceux qui préfèreront une récompense pécuniaire pour avoir arrêté quelques uns de ces criminels, recevront la somme promise dans le décret cité plus haut; mais ceux qui aimeront mieux être élevés en dignité, s'ils sont déjà mandarins passeront à une place plus élevée; si, pour quelque délit, is ont perdu leur dignité, ils la recouvreront, et les simples particuliers seront élevés à la dignité de centenier. De cette manière chacun recevra pour ses mérites, une récompense conforme à ses voeux. Quelle joie ils en éprouveront! et de quelle nouvelle ardeur, ils vont être animés pour rechercher ces coupables; que de nouveaux moyens, que d'habiles stratagèmes ne vont-ils pas inventer pour les arrêter sûcement et les livrer aux mandarins? Dorénavant les prêtres ne sauront plus où fuir pour éviter les châtiments qu'ils méritent, et les Chrétiens sachant ce qu'ils ont à craindre abandonneront bientôt leur mauvaise doctrine.

Nous disons en second lieu, que pour nous prémunir contre les invasions des Cambodgiens, il fitut garder avec soin nos frontières, en les garnissant de forts qui en défendent l'entrée. Les Cambodgiens nous sont tributaires, mais ce sont des barbares qui se réunissent pour marcher ensemble comme les Sangs et les Bois et venir nous susciter de mauvaises affaires $\left.{ }^{1}\right)$. Il est vrai, nos fion-

1) D'après les livres chinois les Sangs sont des espèces de loups qui ont les pieds de devant très longs et ceux de derrière très courts ; e'est tout le contraire pour les Bois; 
tières vers l'ouest sont actuellement très en paix. Mais il est bon de se précautionner pour éviter les malheurs possibles; il vaut mieux arrêter le mal dans son principe que de le láisser éclore, au risque de ne pouvoir ensuite le réprimer; il faut donc envoyer des mandarins pour examiner quels sont les endroits faibles de nos frontières du côté du Cambodge, et y faire élever des forts que l'on garnira de munitions et des troupes avec des barques bien armées, placées partout dans les environs, afin par là d'être prêts à tout évènement fâcheux. Les six provinces qui confinent au Cambodge ont coutume de laisser souvent les soldats en congé; beaucoup d'entre eux désertent; leur service n'est que de dix ans; par conséquent il y a toujours beacoup plus de jeunes soldats que de vieux et peu de bonnes troupes; il faudra donc y prolonger le service des soldats pour avoir le temps de les bien exercer, afin de les rendre habiles et vaillants dans le besoin. Alors nos frontières seront gardées sans peine, et nous serons sans inquiétude sur ce point. Il est aussi très important que les forts que nous éleverons soient munis de vivres en abondance et garnis de bons soldats. Une petite troupe de vaillants guerriers est meilleure qu'une multitude de soldats médiocres. Il faudra autant que possible élever ces forts dans les lieux où il y a de bons terrains à défiicher. On fournira aux soldats d'élite qui seront placés dans ces forts, des bufles, des instruments aratoires, des semences, de l'argent, des vivres et tout ce qui leur sera nécessaire pour les premiers frais de culture de ces terrains, aftn ensnite d'y cueillir du riz pour leur subsistance. $\Lambda$ ux soldats qui voudront s'y établir et y rester définitivement, on donnera en pleine propriéte tout le terrain qu'ils auront défriché; et ils seront à perpétuité exempts de tout impôt. Il est vrai, ces frontières étant des lieux solitaires et lointains, peu de gens veulent s'y fixer pour les cultiver. Mais la position des soldats qui s'y établiront sera incomparablement plus avantageuse que celle des particuliers qui viennent s'y fixer. Ils y seront sous la protection du Gouvernement qui leur fournira l'argent, les vivres et tous les objets qui pourront leur manquer. Ils seront logés dans les forts à l'abri des voleurs et des brigands. Leur service militaire ser'a lare et léger; ils auront beaucoup de temps de reste pour se livrer à l'agriculture qui leur procurera une surabondance de bien-être dont ils jouiront paisiblement. En faisant l'oeuvre commune, ils travailleront aussi pour leur intérêt particulier. Ce sera là une position très avantageuse qui fera leur bonheur. Il n'est pas douteux qu'ils ne la recherchent et la désirent avec empressement. Qu'on mette ce projet à exécution et dans peu d'années on en verra les bons résultats. Ces terrains ne seront plus abandonnés et inutiles; nos soldats auront des vivres en abondance; ils seront riches el vaillants, et nos frontières seront mises par là à l'abri de tout danger.

ces animaux ru la disproportion de leur pied, ne peuvent pas marcher seuls, mais réunis l'un sur l'autre ils marchent très bien avec les pieds de devant de l'un et les pieds de derrière de l'autre. Telle est la noble comparaison employée par nos mandarins pour signifier l'alliance des Français avec les Cambodgiens. (N.T.) 
En troisième lieu, nous disons que pour arrêter les entreprises des barbares d'Europe, il faut multiplier les périls près de nos côtes pour leur ôter la possibilité d'en approcher. Ces barbares sont d'un caractère très-ferme et très patient; les oeuvres qu'ils n'ont pas pû achever, ils les lèguent à leurs descendants pour les conduire à la dernière perfection; les projets qu'ils n'ont pas eu le temps de réaliser, ils les laissent aux ages suivants qui les mènent à bonne fin. Ils n'abandonnent ancune entreprise, et ne se découragent par aucune difficulté. C'est là ce qui doit faire le sujet de notre plus grande inquiétude. Ces barbares vont dans tous les royaumes sans redouter ancune fatigue; ils sondaient les peuples sans regretter aucune dépense; quelle est leur intention en cela, sinon de s'emparer des pays qui les reçoivent, et de les infeste: de leur doctrine dépravée. C'est là le seul but de toutes leurs ruses. Il ne convient donc pas de les laisser s'établir dans notre pays; il faut au contraire leur en fermer strictement l'entrée. Avec ce système de sévère défense, même bien peu de prêtres de la Religion de Jésus osent venir clandestinement ici; quelques uns seulement s'aventurent à avoir des communications secrètes avec les descendants des anćens Chrétiens qu'ils cherchent à instruire pour en faire des Prêtres comme eux. Pour cela, ils leur font des largesses pécuniaires et ils les envoient ensuite dans les villages exhorter les sectateur's de leur mauvaise doctrine, afin de les dominer et de s'en faire des partisans qui leur sont dévoués de coeur et n'obéissent au Roi qu'en apparence. Cependant, il parait que ces Barbares, voyant que leur entrée ici est strictement prohibée, qu'ils peuvent difficilement employer leurs artifices et leur habileté parmi nous, se sont dernièrement tournés ver's les Cambodgiens, chez lesquels ils ont appris qu'ils pourraient plus facilement faire les dupes; ils y enseignent l'art des armes pour gagner la confiance du Roi, et font des largesses au peuple pour se l'attacher; ils y bâtissent des maisons où ils enseignent leur religion que les ignorants embrassent à l'envi '); et voila en quoi consistent leurs plus fines ruses.

Dernièrement ils sont venus à la baie de Tourane avec des navires; ils ont fait semblant de demander la liberté de commerce; mais dans le fond, c'était le moyen de propager sourdement leurs monstrueuses erreurs, qu'ils voulaient avoir; ils s'inquietent peu du commerce; mais sous ce spécieux prétexte, ils veulent avoir la facilité d'enfreindre les lois du royaume. Voila ce qu'il faut attentivement considérer, voila ce dont nous devons nous garder avec soin; tel est le point capital ì observer pour un bon gouvernement tant à l'intérieur qu'à l'extérieur.

Votre Majesté est comme le soleil et la lune qui réchauffent et éclairent près et loin; elle connait les précautions ì prendre à l'intérieur comme à l'extérjeur pour le bon gouvernement des barbares et des peuples civilisés; elle

1) On voit qu'ici les mandarins disent plutôt ce qu'ils s'imaginent que ce qui est la la vérité. (N. 'T.) 
sait que les Barbares d'Europe, sont tous pour la propagation de leur mauvaise religion. Tantôt ils s'en vont, tantôt ils reviennent. Ne prendre aucune mesure de précaution contre eux, serait, certes une bien grande imprudence.... Si nous n'avons pas des moyens supérieurs pour réprimer leurs entreprises criminelles, nous devons au moins tâcher de nous en procurer de passables, si nous laissons ces barbares pénétrer dans nos ports avec leurs navires, il nous sera bien difficile de les en expulser ensuite. Il fatut donc leur en rendre l'entrée impossible; c'est là ce que nous avons de mieux à faire. Jusqu'à présent, on n'a jamais vu les Barbares d'Europe oser pénétrer un peu au loin avec leurs navires dans l'intérieur de nos fleuves. La seule inspection topographique des lieux les arrête. S'ils osaient le faire, nous trouverions facilement, selon les circonstances, les moyens de les arrêter avant qu'ils aient eu le temps de s'avancer au loin dans l'intérieur. Le danger n'est donc pas là; il est dans la baie de Tourane qui par son étendue permet aux navires d'y voguer facilement, et qui par les montagnes dont elle est entourée leur offre un ancrage propice à l'ab:i des flots et des vents. Aussi les Barbares d'Europe osent souvent y pénétrer et y rester longtemps à l'ancre sans tenir aucun compte des défenses de Sa Majesté. De plus, cette baie est près de la route ruyale, près des habitations du peuple; elle est aux flancs de la capitale; elle est donc la clef de ce Royaume: c'est l'endroit le plus exposé aux dangers du dehors. Aussi les Barbares d'Europe désirent beaucoup en avoir la possession. Si nous ne leur en fermons pas l'entrée comment les en chasserons-nous, s'ils ne veulent pas en sortir de bon gré lorsqu'ils y auront pénétré? Ces hommes semblables par leurs moeurs aux chèvres et aux chiens ne peuvent pas être persuadés par le langage de la raison. Leur raison à eux, c'est la voix du canon. Mais dans l'art de faire parler le canon, ils sont extrêmement habiles. Nous ne pouvons pas espérer de les surpasser. Fe plus nos forts peuvent tout au plus défendre l'abordage des terres, et eux se tiennent au loin au milieu de l'eau où ils semblent voler comme des oiseaux de proie qui poursuivent le faible passereau. Comment les atteindre?....

Les choses étant ainsi, que faire? leur livrer bataille? nos soldats seront écrasés sans aucun bon résultat; nous soumettre à eux sans opposer la moindre résistance? Cela ne convient nullement. Il ne faut donc pas faire la paix avec eux; il ne faut pas non plus leur livrer bataille, mais nous tenir sur la défensive; et pour cela nous environner de difficultés et de périls; c'est là ce qui convient le mieux pour nous. Dans le livre Dieh on voit que ce système a été mis en pratique autre fois par les anciens Rois. Ce même système pourra encore maintenant nous préserver contre les agressions des barbares de l'ouest bien plus difficilement [sic] que la Grande Muraille élevée jadis par les Songs n'a mis la Chine à couvert contre les attaques de l'ennemi. Il est vrai l'entrée de la baie de Tourane est large; il parait bien difficile de l'obstruer; néanmoins comme l'eau n'y est pas ti'ès profonde, comme les matériaux nécessaires pour 
cela, se trouvent en abondance sur le versant des montagnes qui s'élèvent de chaque côté, on voit qu'avec du courage et de la bonne volonté, cet ourrage peut se faire, et que sans être facile, il ne sera pas non plus d'une très difficile exécution. Il faut donc charger le grand mandarin de la province de QuangNam de faire au plutôt commencer les travaux qui consisteront en une jetée depuis le bas de l'île Duyen-Tuy où l'eau est peu profonde. Cette jetée s'avancera en traver's de l'entrée de la baie jusqu'aux endroits les plus profonds où l'on accumulera des morceaux de pierre et de terre tirés des flancs de la montagne en face, et qui formeront par leurs replis tortueux comme la figure d'un immense serpent caché sous l'eau. Par là, l'entrće de la baie sera resserrée et son fond sera élevé. On ne laissera qu'un étroit passage à portée des canons de la citadelle hä̈-daï et pour aider cette citadelle à repousser les Navires européens qui s'avanceraient près de la terre où elle est située, on élèvera à sa droite et à sa gauche, plusieurs forts garnis de canons dont la portée mesurée d'avance pourra aller les atteindre. Quand arriveront des navires européens, le gardien de la baie les avertira que l'entrée en est défendue, qu'ils aillent, s'ils le veulent jetter l'ancre au delà de la pointe de l'île Daj̉mà. S'ils s'avancent vers la baie pour faire de l'eau, on du bois, on leur intimera l'ordre de s'arrêter en dehors de la portée du canon, et le gardien leur fournira l'euu et le bois nécessaire; et après un jour ou deux, il leur ordonnera de repartir au plutót. Quant à ceux qui mépriseraient ces avertissements, et qui, faisant les braves, oseraient entrer dans l'intérieur de la baie, comment oseront-ils y rester longtemps quand ils verront l'aspect terrible de nos moyens de défense? et si par une orgueilleuse bravade ils en venaient à cet excès d'audace, les canons de nos forts les auront bientôt mis à la raison. Nous pourrons facilement les prendre et les punir selon leurs mérites. L'ouvrage proposé sera très considérable, les soldats seuls ne suffiront pas pour l'exécuter; alors on louera le peuple de la province pour aider à le faire; et si cela ne suffit pas encore, on y emploiera la population des provinces voisines. Si cette oeuvre ne peut être terminée dans un mois, on y travaillera une année, et même plusieur's années si c'est nécessaire. L'important c'est qu'elle se fasse quels que soient le temps, la peine et les dépenses qu'elle pourra exiger. Après ce travail, l'entrée de la baie se retrécira et son fond s'élèvera de plus en plus. Les canons de nos forts pous la garde de la baie ne porteront plus des coups inutiles; nos soldats dans le besoin auront des ressources pour faire une forte résistance; ils auront un abri pour jouir de quelque repos, car il leur sera facile de garder des lieux de si difficile accès, pour l'ennemi. Les Barbares d'Europe n'oseront plus s'aventurer à venir ici. Par ces travaux la capitale sera grandement renforcée et ennoblie, et les Chrétiens Annamites, n'ayant plus d'espérance d'être secour'us par les Européens, pourront facilement être forcés d'abandonner leur mauvaise religion pour embrasser la bonne. Sans cela, quelles peines et quelles inquiétudes n'auront pas nos soldats pour arrêter les projets des Barbar'es d'Europe contre nous? Il faut donc exécuter ce travail 
sans en désemparer jusqu'à son entière terminaison, dussions-nous y employer plusieurs années; autrement nous ne serons jamais tranquilles. C'est un grand travail qui exigera de fortes dépenses, mais il est absolument nécessaire.

Dixième annće du Règne de Tu Duc le 3 de la 5e lune (c'est-à-dire le 25 Mai 1857).

Cette traduction a été faite à Vinh-tri en Sept. 1857 par le Vicaire Apostolique du Tong-King Oriental, Pierre André Retord, Evèque d'Acanthe.

Il est certain que cette pièce se passe de commentaires.

\section{CHAPITRE XVII. \\ Martyre de Mgr. Diaz.}

Rien de surprenant dans ces circonstances que les persécutions eussent redoublé d'intensité; la première victime fut le vicaire apostolique du Tong-king central, Mgr. DIAZ ${ }^{1}$ ); arrêté la veille de l'Ascension à Bui-chu, avec le maire chrétien de ce village, ce prélat fut jeté en prison «avec la chaine au cou et pendant la uuit les ceps aux pieds jusqu'au 20 juillet, jour où il a eu la tête tranchée pour la foi".

Martyre de Mgr. Diaz.

Dans une lettre adressée ${ }^{2}$ ) au Comte Kleczkowski, Mg. Retord ${ }^{3}$ ) raconte ainsi le martyre de Mgr. Diaz:

«On l’a fait asseoir par terre et comme attaché à une croix derrière le dos.

«On n’a pas permis aux Chrétiens d'approcher pour avoir la consolation de recueillir quelque peu de son sang.

"Après l'exécution on a pioché le lieu arrosé par" son sang et avec un grand appareil de soldats et d'éléphants les Mandarins ont promené son corps dans les rues principales de la ville, pour inspirer la terreur au peuple, ensuite

1) Jose Maria Draz, né le 25 octobre 1818, dans la paroisse de Suegos, évêché de Lugo; novice au collége dominicain d'Ocaña, le 23 sept. 1842; fit profession le 24 sept. 1843; parti de Cadix, 10 Mai 1844; arrive à Manille, 14 sept. 1844; part pour Macao et le 'Tong-king, 2 fév. 1845; sacré érêque de Platea, 26 août 1852; vicaire apostolique du Tong-king Central (chef-lieu Bui-chu, (Nam-dinh), 26 août 1852; exécutć 20 juillet 1857.

2) Mission da l'ung-king occidental, 19 sept. 1857.

3) Pierre André ReTond, de Lyon, vicaire apostolique du Tong-king Occidental (cheflieu So'-kiên (Ha-nôi), évêque d'Acanthe $1832-1858 ; \dagger 22$ octobre 1858 , à Dong bau, au Tong-king. 
on a lié son corps et sa tête ensemble dans un tapis redoublé d'une natte, et on l'a mis dans l'eau du fleuve attaché par une corde à une grande barque mandarine qui s'est dirigée à force de rames vers la mer. Un capitaine était assis près de la corde à laquelle était attaché le corps de Sa Grandeur qui suivait en flottant entre deux eaux, les rameurs avaient la face tournée en avant avec menace de coups de rotin pour ceux qui auraient osé regarder en arrière.

«De sorte que personne n'a pu savoir quand ni dans quel endroit la corde qui trainait le corps du Martyr a été coupée. Le fleuve était alors déjà beaucoup grossi, le courant était rapide, le corps du Martyr n'était pas assez appesanti pour couler à fond, ni assez léger pour flotter à vue d'oeil: d'où il est arrivé que malgré les efforts qu'ont faits nos pécheurs et ceux du Tongking central, il n'a pas été possible de le retrouver.

«C'est là, M. le Comte, tout ce que je sais au sujet de l'affaire de Mgr. Diaz; mais cette triste affaire n'est pas la seule que nous ait occasionnée la malheureuse expédition de $M$. de Montigny.

«En Cochinchine tout a été bouleversé; les colléges dispersés, les maisons des missionnaires, des prêtres indigènes et des religieux détruites, plusieurs villages bloqués, beancoup d'effets religieux saisis, environ 80 Chrétiens mandarins ou simples particuliers ont été arrêtés et sournis aux plus terribles tortures, plusieurs vaincus par les tourments ont eu la faiblesse d'apostasier et de fouler la croix aux pieds, et cela ne suffisait pas: on les forçait encore à brûler de l'encens aux idoles en amende honorable pour le sacrilége qu'avaient commis les Français, disait-on, en mangeant et s'asseyant sur un autel consacré aux idoles.

"Cependant il y a eu environ trente de ces Chrétiens qui se sont montrés supérieurs à tous les supplices, et, qui ont été condamnés à un exil perpétuel aux extrémités du Tong-king. La moitié environ de ces confesseurs de la Foi sont déjà arrivés ici, parmi eux il y a 13 soldats appartenant à la garde de la reine mère, et deux femmes dont l'une est une jeune fille de 20 ans, soeur d'un prêtre indigène.

«Dans le Tong-king méridional, il n'y a pas el d'arrestation mais seulement beaucoup de trouble, de frayeur, de dispersion d'élèves, de destruction de maisons, etc. Dans notre Tong-king Occidental nous avons eu aussi beaucoup de mal.

«Nos Chrétiens dans un grand nombre d'endroits ont été impliqués de superstitions, nous avons fait des dépenses pécuniaires considérables pour diminuer les vexations des Mandarins: ni nous, ni les prêtres annamites n'avons pu nous livrer ì l'administration des Chrétiens, parce que nous avons été et nous sommes encore obligés de nous tenir cachés.

«Deux de nos plus gros villages chrétiens ont été bloqués par les Mandarins pour nous prendre. Un de nos prêtres tong-kinois a été arrêté, ainsi que six chrétiens et quatre Catéchistes. 
“Le prêtre nommé Paul Tigne a été mis à mort pour la foi le 6 Avril, deux Chrétiens ont été envoyés en exil, un autre a été condamné à mort avec sursis, un quatrième est condamné à être soldat toute sa vie, un autre aux travaux forcés pendant trois ans, et un autre qui était chef de canton à pe:dre sa place.

«Le 25 mai, un de nos catéchistes a eu la tête tranchée pour la foi dans la province de Doài: deux de nos églises ont été abattues par le mandarin avec la force armée, et près de la moitiẻ de notre bel établissement de Vignetri où nous avions près de 200 élèves a été détruit, et la moitié de nos élèves dispersés.

"Ce que nous avons pu conserver ne l'a été qu'au prix d'une bonne somme d'argent donnée .aux mandarins."

Martyre de

Parmi les Chrétiens qui furent arrêtés à l'occasion de la venue Ho Đinh-tri.

des Français se trouvait un haut fonctionnaire de la capitale Ho Đinh-tri, qui après de cruelles tortures fut mis à mort. Voici le récit de Mgr. Sohier:

Messeigneurs les Evèques et Messieurs les Missionnaires du Tong-king. Messeigneurs et très chers confrères.

«Peut-être qu'à la fin, je finirai par vous ennuyer par mes angéliques. “Cependant je crois qu'il est bon de vous en adresser encore une au sujet de «la bienheureuse mort du grand mandarin thài-bọe. Vous savez déjà que «d'après une requête du tribunal appelé Đu-sàt-vien (espèce de tribunal de «police), il fut arrêté le 8 novembre; le jour suivant, il fut mis à la question «et on le frappa durement. La crainte et la violence des tortures lui arrachèrent «des aveux mélés de mensonges qui ont attiré sur nous toutes sortes de mal"heurs; il dit qu'il connaissait un prêtre nommé boi; indiqua le lieu de sa «naissance et celui de sa résidence; que ce Prêtre faisait tous les ans le «catalogue des Chrétiens à la suite du dernier édit de persécution; ce prêtre «avait écrit en France pour implorer du secours; qu'il avait confié son fils au «prêtre oai pour l'instruire et que de son consentement, celui-ci l'avait envoyé «étudier à Singapore (à Pinang) auprès de Mgr. Lefêvre. Que le P. oai avait «reçu une lettre de Mgr. Miche qui lui apprenait que des navires français «devaient venir demander la liberté de religion et enfin comme on lui demanda «'il connaissait d'autres mandarins chrétiens, il en déclara cinq et quelques «uns de ses parents etc. Mais ce Mandarin a toujours refusé dapostasier quoi «qu'on le lui ait ordonné bien des foỉ. Depuis il s'est grandement repenti de «ce qu'il avait dit et versé bien des larmes pour expier son péché, cependant "toutes ces déclarations allumèrent un incendie épouvantable dans notre mission; «un grand nombre de Chrétiens furent arrêtés, jettés en prison et roués de 
kroups de rotin. On leur demandait surtout des renseignements sur le P. odi «et le fils du grand Thài-boc. Mais tous répondirent qu'ils ne savaient rien; «ensuite on leur ordonna de profaner la croix. Quelques uns, mauvais Chrétiens, «ont eu le malheur de le faire. Mais on ne s'est pas contenté de leur faire "fouler la croix aux pieds. On les conduisait ensuite dans les pagodes et les «temples des idoles, pour leur faire saluer le démon et les forcer ì leur offrir «de l'encens. Le 6 mars, le mandarin Thàj bộc reçut six coups de rotin et le «9 mars dix coups; on lui demanda s'il avait des relations avec les navires «européens; il répondit qu'il était allé quelques fois rendre visite au P. oai qui «lui avait parlé des navires européens, mais qu'il n'avait aucune intelligence «avec les Européens. Ensuite les Mandarins lui ordonnèrent de déclarer les "prêtres des autres provinces qu'il connaissait. Il répondit, je demeure dans la "province de la Capitale, je ne connais que cette province, je ne sais ce qui se "passe dans les autres. Les Mandarins ne se contentèrent point de ces réponses, «ils lui ordonnèrent de faire une nouvelle déclaration par écrit, jointe à celle «qu'il avait déjà faite auparavant. Mais il leur livra seulement la première sans "y faire presque aucun changement. Car les Mandarins avaient ajouté une foi «entière à ce qu'il leur avait dit d'abord, et il était impossible de revenir là«dessus sans s'exposer à des tortures horribles sans espérance d'obtenir un bon «résultat. Cependant les Mandarins voyant que malgré toutes les tortures ils «ne pouvaient venir à bout de découvrir le P. Oai et n'obtenant aucun nouvel «aveu, s'occupèrent de rédiger la sentence des prisonniers. Nous n'avons pu «encore nous la procurer parce qu'on exige pour cela une somme trop consi"dérable, mais j'espère que nous l'aurons bientôt Nous avons pu obtenir seu«lement la sanction royale dont voici la traduction française ( $\mathrm{Mgr}$. Sohier nous «"envoie en langue annamite).

Décret royal.

Le Sieur Ho-tinh-hi' ${ }^{1}$ ) était un simple greffier; de grades en grades, il est monté jusqu'à la dignité de grand mandarin; il a eu l'audace de mépriser nos défenses en suivant une Religion mensongère, sans jamais s'en corriger en aucune manière. Bien plus il a envoyé son fils étudier à Singapore; il s'est liè d'amitié avec le prêtre Oai. Il est clair que c'est un ingrat et un servileur infidéle dont le coeur est partagé. Qui pourrait énumérer tous ses crimes? il faut donc le punir selon la loi pour l'exemple des autres Chrétiens, et leur apprendre à s'amender.

"Mais avant l'exécution, le Roi ordonna de le conduire trois fois dans les «places publiques de la ville et des environs, et de lui donner chaque fois, «trente coups de baton, après qu'un hérault aurait publié en chinois et en «annamite l'ordonnance suivante:

1) C'est le nom propre de ce mandarin: Thài-boe est le nom de sa dignité de chef des tailleurs royaux, gardien en chef de la garde-robe. 
Far ordre du Roi, deux Mandarins du Conseil intérieur, un capitaine du tran phat, et dix soldats, doivent pendant trois jours conduire le Sieur Ho-dinh-i, une fois chaque jour. dans toutes les places de la ville et dans lous les marchés d̀ l'extéricur des forlifications, un jour dans tels ou tels lieux, un autre jour dans tels ou tels autres pour y publier ses crimes comme il suit:

C'est un sectateur d'une religion perverse; il s'apprêtait à la révolte: un ingrat, un imposteur des lois, qui a oublié les bienfaits dont le Roi l'avait comblé; les Chrétiens disent que ceux qui pour leur Religion souffrent ainsi les tourments et la mort, vont au Ciel: qui sait si cela est vrai? Mais pendant qu'ils souffrent des tourments si atroces où donc est leur Jésus? pourquoi ne vient-il pas les délivrer? n'est-il pas clair d'après cela qu'il n'y a aucune utilité dans l'observation de cette Religion? voila ce qu'il faudra publier pendant trois jours, afin que tous le sachent et tremblent.

Aprés la publication des paroles susdites, on lui administrera 30 coups de baton; on l'exécutera publiquement afin que tous les Chréliens le voient, ayant honle de leurs erreurs et s'en corrigent.

"Cet édit a été punctuellement exécuté par trois fois. La première fois qu'on wa conduit ainsi le mandarin Michel Hô-đinh-hi, bon nombre de Chrétiens le «recevaient en pleurant en se lamentant. Mais quand il fut revenu dans sa «prison il les gronda et leur défendit de faire de pareilles démonstrations de "crainte d'être compromis à cause de lui. C'est le 22 mai qu'on le condnisait «au supplice, il marcha d'un pas rapide pendant $1 / 2$ heure ou $3 / 4$ d'heure, «portant sa chaine au milieu de quatre soldats l'épće nue: un cavalier le «précédait, cinq autres le suivaient. De chaque côté, il y avait une haie de «soldats; une multitude de Chrétiens et de païens étaient accourus à ce spectacle, «les uns par curiosité, les autres pour lui donner une dernière marque d'affection «et de tendresse. Mais les Chrétiens comprimaient leurs larmes, car il y avait «des ordres secrets pour arrêter ses parents et ses amis. Tous ses anciens «serviteurs lui témoignèrent un grand dévouement et l'assistèrent jusqu'an «dernier moment. Deux prêtres le suivaient confondus dans la foule et lui «donnèrent plusieurs fois l'absolution, comme ils en étaient convenus d'avance. «Pour lui il priait continueliement et ne montra aucune faiblesse. Il était revêtu, «d'habits simples et pauvres comme il avait toujours fait depuis son arrestation «par esprit d'humilité et de pénitence; quand il fut arrivé sur le tapis et les «toiles qu'on avait préparés dans le lieu du supplice, il se lava les pieds, fûma «une dernière pipe; ensuite il se mit à genoux, disposa lui-même ses cheveux «et ses habits; puis au signal donné, un bourreau lui a abattu la tête de «deux coups de sabre; il était vers onze heures. Son corps fut enterré dans la «soirée à Phì-càm.

«Un instant avant d'être exécuté, il présenta au Mandarin qui l'accom«pagnait nne nouvelle déclaration qu'il avait préparée, où il rétractait les 
«mensonges qu'il avait faits sur le compte du $P$. Oai, mais aucun mandarin «ne voulût s'en charger et il la laissa à côté de lui, du reste il avait plusieurs "fois demandé pardon à ses compagnons de prison qui avaient été arrêtés à «cause de lui, et s'était prosterné devant eux et quand j'appris que sa sentence «était terminée, je lui écrivis de ne plus s'occuper de sa nouvelle déclaration, "parceque c'était peine inutile. Quelques jours avant sa mort, un prêtre lui "porta la $S^{\text {to }}$ Communion en prison et il s’était confessé plusieurs fois; en un «mot, il a fait la mort la plus édifiante, et j'espère qu'un jour l'Église le "comptera au nombre de ses martyrs. Quant aux autres confesseurs qui ont "refusé d'apostasier ils sont tous condamnés à l'exil dans cinq provinces diffé«rentes à l'extrémité du Tong-King. Nous ignorons encore quand ils partiront «et je n'en connais pas le nombre au juste, parce qu'il y a quelques vieillards «que l'on a promis de racheter à prix d'argent.

«Il y a ordre de rechercher et de poursuivre le prêtre Oaï pendant six «mois, ainsi que sa mère, sa femme et le fils de Thài-bôe et quatre autres «personnes. Après l'exécution du Thài-bôe, le Roi a publić un édit oú il gronde «les Mandarins de la ville et des provinces de ce qu'ils ne sont pas assez «sincères [sévères?], et n'exercent pas la surveillance assez sévèrement, etc., etc. “Cet édit ne contient que des généralités et ne parle point de religion en "particulier. Mais on m'assure que le Roi a donné ordre d'en fabriquer un «autre pour abolir le nom chrétien.... Je suis menacé de la visite du gouver«neur Thùà-thien et Quảng-tri; hier on m'en a porté la nouvelle. Si vous en «avez l'occasion je vous prie d'envoyer les nouvelles à Hong-kong ou à Macao «afin que les Français connaissant notre détresse, se hâtent de venir à notre usecours. C'est dans cette espérance que j'ai l'honnem d'ŝtre, etc.

Joseph, Evêque, de Gadara et Coady.

Voici encore ce que dit Mgr. SoHır ${ }^{1}$ ) dans une lettre parti- Lettre de culière du 6 juillet:

"Nous avons grandement besoin d'être promptement secourus car nos affaires "s'embrouillent de plus en plus. Les mandarins, chefs de huyên et les chefs de «cantons, continuent de vexer nos Chrétiens à outrance, surtout à la capitale.

«ll y a des endroits où tous les Chrétiens abandonnent leurs maisons pour «se sauver oì ils peuvent, de crainte d'être arrêtés par les mandarins. Le Roi «a ordonné de mettre à la question les gens de thae-hön pour savoir au juste «à qui ils voulaient livrer le catalogue qui fut pris par des païens après la «Toussaint. (C'était un Catalogue des morts dans cette Chrétienté qu'on recom-

1) .7oseph Hyacinthe SoHıer, du diocèse du Mans; Missions étrangères de Paris; parti le 21 déc. 1842; érêque de Gadara, coadjuteur en Cochinchine septentrionale en 1851; vicaire apostolique en 1862 ; † à Ke-sen (Quang-binh), le 3 sept. 1876, à 58 ans. 
«mandait alıx prières des Chrétiens le jour de la commémoration des morts. «Le Roi croit que c'est un Catalogue des guerriers qu'on devait fournir aux «(Françajs). Grand nombre des hommes de cette Chrétienté ont été arrêtés. «C'est là une grosse affaire qui n'est pas encore terminée. Quatorze soldats «chrétiens de la garde de la Reine mère ont été arrêtés, un d'eux a apostasié, «les autres tiennent ferme jusqu'à présent. Un mandarin nommé Nguyên đin«trü vient de présenter au Röi un nouveau projet d'Edit contre la Religion. "Le roi l'a beaucoup loué de son zèle; il a livré ce projet aux grands mandarins «de la capitale pour être examiné et voila tout ce que renferment les lettres «de Cochinchine.»

Dans le Tong-king, depuis le martyre de Mgr. Diaz, voici en abrégé ce qui est arrivé de nouveau: un de nos Chrétiens nommé Nhiế mi, marchand de Khoang a été arrêté pour cause de religion et comme il a été très-ferme dans sa foi, et n'a jamais voulu fouler la croix aux pieds, il a été arrêté condamné à l'exil dans la province de Lao Bońg. Nous avons maintenant une inondation qui est dans certains endroits plus forte que celle de l'année dernière. - Toute la moisson du $10^{\text {ene }}$ mois qui était très belle est entièrement perdue et comme celle de la même saison de l'année dernière fut détruite aussi par l'inondation et que celle du $5^{\mathrm{e}}$ mois a été très mauvaise à cause de la sécheresse, jugez de la famine qui doit régner et régnera ici jusqu'à la moisson du $5^{\mathrm{e}}$ mois de l'année prochaine. Rien ne peut s'imaginer de plus lamentable. Le jour de l'Assomption nous avons eu une tempête qui a causé de l'inondation a fait, surtout dans la province de hò-nội un mal incalculable, car beaucoup de maisons ont été détruites et beaucoup de gens sont morts noyés.

(Sig.) + Pierre Evêque d'Acanthe.

Après le supplice de Ho-dinh-tri (22 mai 1857) la situation des Chrétiens était deveuue terrible: «Les prisons regorgeaient de chrétiens, les missionnaires se cachaient où ils pouvaient. Mgr. Cuenot ${ }^{1}$ ) s'abritait à Go-thi; son provicaire, M. Herrangt ${ }^{2}$ ), fuyait de paroisse

1) Etienne Théodore Cúx́xor, de Besançon; Missions étrangères de Paris; parti le 27 janvier 1828; évêque de Métellopolis, coadjuteur, 1835; vicaire apostolique de la Cochinchine Orientale (chef-lieu, Qui-nhon) 1840; mort en prison au Binh-dinh, le 14 nov. 1861 .

2) Charles Ferdinand Herrungt, du diocèse de Cambrai; Missions ét. de Paris; parti le 19 avril 1853; missionnaire de lo Cochinchine orientale; provicaire apostolique; t à Saigon, le 20 jain 1863, a 46 ans. 
en paroisse; Mgr. Lefebvre ${ }^{1}$ ) était aux environs de Saïgon, recueilli par des fidèles qui bravaient la mort pour le sauver, tandis que ses missionnaires, M. Borelle ${ }^{2}$ ) et M. Pernot ${ }^{3}$ ), arec quelques séminaristes, cherchaient un refuge dans les provinces de l'Ouest et dans les iles du Mekong» ${ }^{4}$ ).

\section{CHAPITRE XVIII.}

\section{Intervention de M. de Bourboulon.}

Dès que la nouvelle de l'arrestation de Mgr. Diaz fut parvenue Action de M. à Macao, M. Nicasio Cañete y Morat, Consul Général de Sa Ma- lon. jesté Catholique, s'adressa à notre Ministre, M. de Bourboulon, lui demandant en l'absence de tout bâtiment de guerre espagnol dont il put disposer, de faire envoyer un navire de guerre français sur les côtes du Tong-king pour réclamer la délivrance de Mgr. Diaz; sans perdre de temps, notre Ministre pensant «que tant au point de vue d'humanité qu'en raison de tous les liens d'amitié et de sympathie qui unissent la France à l'Espagne, il ne pouvait qu'entrer daus les intentions du Gouvernement de S. M. Impériale que nous prêtassions au représentant et aux Missionnaires de ce pays le secours qu'ils sollicitaient de nous dans un intérêt si pressant» dépècha le Comte $\mathrm{K}_{\text {LeczorowskI }}{ }^{5}$ ) avec une lettre officielle (31 Août

1) Dominique Lepervre, du diocèse de Bayeus; Miss. èt. de Paris; parti le 15 mars 1835 ; coadj. de Cochinchine, évêque d’lsanropolis, 1842; premier vicaire apostolique de la Cochinchine Occidentale (cheflieu, Saigon) en 1844: démissionnaire en 1864; † à Marseille en rentrant en France, le 30 avril 1865, à l'age de 55 ans.

2) Jean Pierre Henri Marie Bonelue, du diocèse de Toulouse; Miss. ét. de Paris; parti le 27 février 1846; missionnaire en Cochinchine occidentale; provicaire; +15 juillet 1860 , ̀̀ 40 ans.

3) Jean Claude Pernor, du diocèse de Besançon; Miss. ét. de Paris; parti 4 sept. 1852; missionnaire de la Cochinchine occidentale; député des missions de la Cochinchine au séminaire de Paris, 1861.

4) Hist. gén. de la Soc. des Missions ét. par Adrien Launay, III, 1894; pp. 372-3.

5) Michel Alexandre, comte KLeczkowskr, né le 27 février 1818, au château de Kleczkow, en Gallicie; attaché au consulat de Chang-Haï, 19 mars 1847; naturalisé fran- 
1857) vers l'amiral Rigaudt de Genovilly ${ }^{1}$ ), alors mouillé à Castle Peak pour expliquer à cet officier général ce dont il s'agissait et le prier, s'il partageait sa manière de voir, d'accéder à la demande du Consul géneral d'Espagne. Dès le 2 Septembre, l'Amiral répondait à M. de Bourboulon que malgré l'arrivée prochaine du Baron Gros $^{2}$ ) et le besoin de concentrer ses forces il expédierait sur le champ le Catinat dans le Golfe du Tong-king, vers les parages les plus rapprochés de l'endroit où l'évêque espagnol avait été emprisonné, pour réclamer sa liberté et si possible le ramener à Macao. Le caractère de la mission devant être d'ailleurs tout pacifique, il fut convenu que le Comte Kleczkowski serait chargé non seulement de la partie diplomatique, mais encore de la direction générale de l'entreprise, aussi reçut-il de M. de Bourboulon les instructions suivantes:

Macao, le 2 septembre 1857.

Monsieur.

Instructions

à M. Klecz kowski.
D'après les intentions que je vous ai déja fait connaître et ce qui a ćté arrêté entre M. l'Amiral Rigault de Genoullis et vous, relativement à la mission que vous allez remplir avec le concours de la corvette à vapeur de S. M. I. le Catinat sur les côtes de la Cochinchine, je n'ai pas à entrer ici dans de longues explications à ce sujet, mais me bornerai à résumer brièvement l'objet de cette mission, et lá manière dont vous devrez procéder, sauf ce qui ne peut être prévu, pour tâcher de la mener à bonne fin:

«Le but étant, s'il en est encore temps, de retirer Mgr. Diaz de la position critique où il se trouvait à la date des dernières nouvelles reçues par la procure espagnole, et comme pour cela il est de la plus grande importance que le

çais en 1850; attaché payé à la légation de France, Peking, 1854; chargé d’affaires, ler Juin 1862 au 11 avril 1863, puis secrétaire-interprête pour la langue chinoise à Paris. Chargé d'un cours libre de chinois pratique, il fut nommé professeur à l’ḱcole des Langues orientales vivantes à la fin de 1871 ; il est mort le 23 mars 1886. - Cf. Henri Condier, Relat. de la Chine, I, p. 131, note.

1) Charles Rigault de Genoulluy, né à Rochefort (Charente inférieure) le 12 avril 1807; † le 4 mai 1873; contre-amiral, 2 déc. 1854; vice-amiral 9 aout 1858.

2) Le Baron Gros, notre ambassadeur en Chine, arriva le 13 octobre 1857, sur l'Audacieuse au monillage de Castle Peak Bay, ou était ancrée l'escadre de l'amiral Rigault de Genouilly. - Cf. Henri Cordira, L'Expédition de Chine de 1857-58. 
secours que nous voulons lui porter arrive le plus promptement possible, vous vous dirigerez avec le Catinat sur le point de la côte du Tong-king le plus rapproché du lieu de sa détention où ce bâtiment pourra aborder. Là vous ferez connaître l'objet de votre mission aux autorités du littoral et tacherez de faire parvenir par leur intermédiaire au préfet de Nann-Ting, et aux autorités le la province une réclamation tendant à ce que l'Evêque soit immédiatement tiré de sa captivité et amené à bord du Catinat.

«Dans le cas où toutes vos démarches pour obtenir la délivrance de Mgr. Diaz des autorités de la province seraient infructuenses, et où elles vous renverraient à présenter votre demande au Gouvernement Annamite lui-même, après vous être convaincu de l'inutilité d'un plus long séjour sur la côte du Tong-king, vous vous rendriez à Tourane et adresseriez de là à la Cour de Hué une demande instante pour que ce prélat vous soit délivré. - En cas de refus définitif de la part de celle-çi, vous vous borneriez à lui adresser une protestation énergique contre l'indigne traitement et la captivité infligés à un prélat catholique appartenant à une nation amie de la France, et à la rendre responsable de son sort ultérieur.

«Dans le cas qui n'est que trop à prévoir où, en arrivant sur la côte du Tong-king à proximité de la ville de Nann-tinn vous apprendriez que le malheureux évêque a été mis à mort, après avoir recueilli toutes les informations possibles sur les circonstances de sa captivité et de sa mort, vous vous rendriez également avec le Catinat à l'entrée de la riviére de Hué, et feriez parvenir au Gouvernement annamite une note dans laquelle vous protesteriez en mon nom contre ce nouvel attentat et cet acte de barbarie commis sur un missionnaire catholique, diriez que c'est un nouveau grief ajouté à tous ceux dont il a déjà donné sujet à la France, et lui feriez entendre que le Gouvernement de l'Empereur viendra probablement avant longtemps lui en demander un compte sévère.

«Il est bien entendu du reste que votre mission devra se borner absolument à cet objet, à réclamer la mise en liberté du Vicaire apostolique du Tong-king central, que vous n'emploierez à cet effet aucune menace, et que le Catinat ne devra faire usage de ses canons que dans le cas d'insulte ou de légitime défense.

«Il ne me reste qu'à faire des voeux pour le bon succés de cette mission, dans laquelle je sais que vous apporterez tout le zèle et toute l'habileté qui peuvent la mener à bien, et pour laquelle je suis persuadé aussi que vous trouverez dans M. Je Commandant du Calinat le concour's le plus empressé et le plus efficace.

Recevez, etc.

Sig. A. Bourboulon.

D'autre part, l'Amiral Rigault de Genouilly donnait également des instructions au commandant du Catinat: 
L'Amiral

Rigault de Genouilly au Commandant du Catinat.

Frégate Némésis, le 2 septembre 1857.

Monsieur le Comrnandant,

"Vous allez conduire dans le golfe du Tong-king M. le Comte KLEczkowski, Secrétaire de la Légation de France, chargé d'une mission spéciale dont j'ai arrêté les dispositions de concert avec $M$. le Ministre. Cette mission tout-à-fait pacifique, dépouillée de tout caractère agressif, a pour but principal d'obtenir la délivrance de Mgr. Diaz, missionnaire espagnol, vicaire apostolique du Tongking central, arrêté et mis en prison dans la ville de Namting. Vous éviterez avec soin toute mesure qui pourrait amener des hostilités, - je vous recommande à cet égard la plus grande prudence; le moment n'est point encore venu de régler nos comptes avec l'Empire d'Annam. Il appartient essentiellement à $M$. le Baron Gros de le fixer, et son initiative à cet égard doit rester complètement libre. Vous aurez, M. le Commandant, à obtempérer à toutes les les réquisitions de M. le Comte Kleczkowski tontes les fois qu'elles ne vous paraîtront pas de nature à compromettre la sureté de votre bâtiment, ou à entrainer comme conséquence des mesures hostiles. La force ne devra être employée que pour repousser la force et je ne doute pas que, dans ce cas, l'épreuve ne tourne à l'honneur du Catinat. Comme l'arrestation de Mgr. Diaz n'est qu'un épisode de la persécution générale, qui sévit au Tong-king contre les Chrétiens, il est entendu que votre bâtiment donnera asile à tous les missionnaires ou chrétiens indigènes qui viendraient chercher un refuge sous votre pavillon.

«En quittant Macao vous dirigerez votre route de manière à suivre de près les côtes sud de l'ile d'Hai-nan. Il importe que vous ménagiez la ressource des relaches que présentent ces côtes, en cas de mauvais temps, non pour le Catinat, mais pour l'aviso à vapeur à faible tirant d'eau le Lily, que M. le Consul Général d'Espagne a frêté pour vous accompagner dans cette expédition. Vous garderez autant que possible cet aviso à la remorque, jusqu'à ce que vous ayez l'occasion d'utiliser ses services; je recommande la sécurité de ce navire à toute votre sollicitude.

«De l'extrêmité sud d'Hai-nan, vous viendrez attérir sur l'Isle du Tigre (carte de partie de la cóte de Cochinchine, voyage de la corvette la Favorite, 1831). A partir de l'isle du Tigre, le Catinat remontera dans le golfe du Tongking, en suivant la route tenue par la Favorite, dont vous vérifierez les sondes, puis viendra s'arrêter au Cap Boung-Quiona en un mouillage exploré par cette corvette. C'est au bourg situé sur cette rade que M. le Cte. Kleczkowski entrera d'abord en relation avec les autorités du pays; il est présumable que ces relations amèneront la solution désirée, et, dans ce cas, dès qu'elle sera obtenue, vous ferez toute hâte, pour rallier Macao d'abord, et peu après la frégate la Némésis. Si le cas contraire se présentait, peut-être seriez-vous obligé de vous porter plus près de la ville de Namting, située elle-même non loin de la cité de Kehoa, marquée sur les cartes à proximité de l'Isle de Tsin-gay. 
Cette partie de la côte a été parcourue en 1849 par le brig de S. M. B. la Columbine, toutefois conme cette exploration est restée inédite, vous entourerez votre navigation dans ce parage des précautions les plus minutieuses. Si les tentatives nouvelles faites à Kihoa n'aboutissent pas, il pourrait devenir nécessaire que votre Bâtiment se portât plus au Nord, pour s'approcher de la capitale du Tong-king, et dans cette hypothèse, en raison des rivières qui débouchent dans la partie N. O. du golfe et qui projettent des bancs au large, vous redoublerez de précautions pour assurer la sécurité de votre navigation.

«Du moment où vous serez arrivé sur les côtes de Cochinchine près de I'Isle du Tigre, vous placerez à bord du Lily un officier de confiance avec 25 ou 30 hommes, armés de carabines et largement pourvus de munitions. Cet officier ne prendra pas le commandement de cet aviso qui doit rester exclusivement aux mains du Capitaine responsable. Son rôle sera de surveiller les sondeurs, de correspondre avec vous, d'assurer la garde et la défense du navire en cas de besoin. Dès que votre détachement sera à bord, le Lily arborera le pavillon Français. Sous auıun prétexte cet aviso ne doit s'éloigner du Calinat, de façon à être privé de la protection de la corvette.

Je crois, M. le Commandant, qu'en vous donnant la mission importante et honorable qui vous est confiéé, j'ai pourvu à tous les moyens d'en assurer le succés. A vous d'user de ces moyens; je compte sur tout votre zèle et sur tout votre dévouement pour le service de l'Empereur. Vous vous rappellerez que l'Empereur est le protecteur des chrétientés catholiques dans cet ExtrêmeOrient, et que, tout ce qui valide ce titre, ne peut manquer d'être accueilli avec faveur par S. M. Dès votre retour, vous me remettrez un rapport circonstancié, sur vos opérations.

Recevez, etc.

Sig. Rigault de Genouilly.

Comme il était nécessaire aussi que le Catinat fut accompagné d'un navire à vapeur d'un plus faible tirant d'eau que lui, soit pour éclairer sa marche aux approches des côtes, soit pour transporter le Secrétaire de la Légation de France plus à proximité des endroits où il pourrait se mettre en rapports avec les autorités tonquinoises, et nos canonnières même calant encore trop pour pouvoir remplir cet objet, le Cousul Général d'Espague ne voulant rien épargner pour tâcher de sauver la tête du vénérable prélat, affrêta moyennaut la somme de 5000 piastres un excellent steamer de Commerce appelé le Lily, appartenant à un négociant américain 
de Macao, qui fut mis au service de la mission et placé sous la protection du Catinat.

Isttre de $M$. «Tous les soins, écrit 1) M. de Bourboulon, au Ministre des Affaires Etrangères, de Bourboulon ont été pris pour circonscrire cette expédition dans les limites d'une énergique des Affaires réclamation à l'effet de sauver, s'il en est encore temps, la vie et d'obtenir la Etrangères. liberté du missionnaire espagnol, et pour lui ménager le plus de chance possible de succès.

«A part l'intérêt qui s'attache naturellement à l'objet spécial de la mission du Catinat, l'évènement qui l'a motivée me parait emprunter pour nous une importance particulière à la position dans laquelle nous nous trouvons aujourd'hui placés vis-à-vis du gouvernement annamite en raison de la manière dont s'est terminée la mission de M. de Montigny. On ne saurait se dissimuler que l'arrestation de Mgr. Diaz n'est point un fait isolé, mais un incident de la persécution qui même pendant la présence de nos båtiments de guerre à Tourane avait sévi avec rigueur contre nos missionnaires et leurs chrétientés et que tout fait présumer avoir pris un nouveau degré d'intensité depuis leur départ. Le sort qui aura été fait à l'évêque espagnol, la décision qui aura été prise par la Cour de Hué sur la question de vie ou de mort qui lui avait été déférée, sera donc l'indice le plus significatif et nous donnera la mesure exacte des dispositions dans lesquelles elle est restée à notre égard, car si par malheur Mgr. Diaz avait été exécuté, ce serait la preuve que le Gouvernement Annamite enhardi par le dernier succès remporté sur nous, a pris le parti de mettre de côté tous ménagements, et nous devrions nous attendre à apprendre d'un moment à l'autre qu'il a fait subir le même sort à l'un de nos missionnaires. Sous ce rapport, M. le Comte, la mission du Catinat, surtout si elle arrive encore à temps pour prévenir le dénouement sanglant qui n'est que trop à craindre, peut exercer une influence salutaire sur les dispositions et la conduite du Gouvernement Cochinchinois, en lui montrant que si nous nous sommes retirés momentanément, nous n'avons pas cessé pour cela d'observer et de surveiller ses actes, le contenir dans de certaines bornes de modération et épargner à la France d'avoir à déplorer de nouveaux malheurs et à venger de nouvelles insultes».

1) Macao, 8 sept. 1857 .

(à Suivre). 